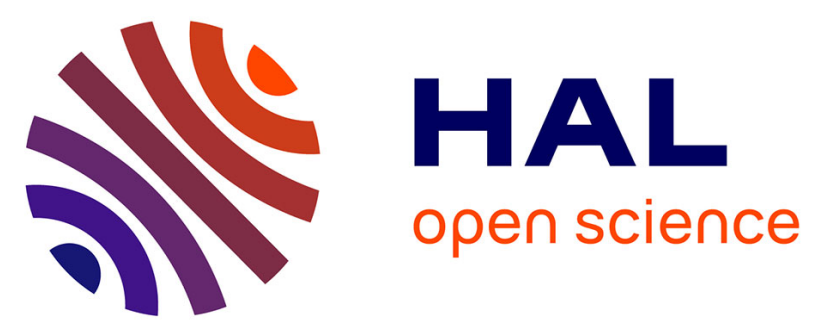

\title{
Heritability estimates from genomewide relatedness matrices in wild populations: Application to a passerine, using a small sample size \\ C. Perrier, B. Delahaie, A. Charmantier
}

\section{- To cite this version:}

C. Perrier, B. Delahaie, A. Charmantier. Heritability estimates from genomewide relatedness matrices in wild populations: Application to a passerine, using a small sample size. Molecular Ecology Resources, 2018, 18 (4), pp.838-853. 10.1111/1755-0998.12886 . hal-02326729

\section{HAL Id: hal-02326729 \\ https://hal.science/hal-02326729}

Submitted on 22 Oct 2019

HAL is a multi-disciplinary open access archive for the deposit and dissemination of scientific research documents, whether they are published or not. The documents may come from teaching and research institutions in France or abroad, or from public or private research centers.
L'archive ouverte pluridisciplinaire HAL, est destinée au dépôt et à la diffusion de documents scientifiques de niveau recherche, publiés ou non, émanant des établissements d'enseignement et de recherche français ou étrangers, des laboratoires publics ou privés. 
1 Heritability estimates from genome wide relatedness matrices in wild populations:

2 application to a passerine, using a small sample size

3

4 Running head: GRM in Corsican blue tits

5

6 Perrier $\mathrm{C}^{*}$, Delahaie B, Charmantier A.

7

8 Centre d'Ecologie Fonctionnelle et Evolutive, CNRS-UMR5175 CEFE, Montpellier,

9 France

10

$11 \quad{ }^{*}$ corresponding author: charles.perrier@cefe.cnrs.fr

12

13 Molecular Ecology Resources - Special issue Association Mapping in Natural

14 Populations.

15

16 Keywords: heritability; genetic correlation; RAD-sequencing; GRM; pedigree;

17 partitioning; phenotype; blue tit; SNP; Cyanistes caeruleus 


\section{Abstract}

Genomic developments have empowered the investigation of heritability in wild populations directly from genome wide relatedness matrices (GRM). Such GRM based approaches can in particular be used to improve or substitute approaches based on social pedigree (PED-social). However, measuring heritability from GRM in the wild has not been widely applied yet, especially using small samples and in non-model species. Here, we estimated heritability for four quantitative traits (tarsus length, wing length, bill length and body mass), using PED-social, a pedigree corrected by genetic data (PEDcorrected) and a GRM from a small sample $(n=494)$ of blue tits from natural populations in Corsica genotyped at nearly 50,000 filtered SNPs derived from RAD-seq. We also measured genetic correlations among traits and we performed chromosome partitioning. Heritability estimates were slightly higher when using GRM compared to PED-social, and PED-corrected yielded intermediate values, suggesting a minor underestimation of heritability in PED-social due to incorrect pedigree links, including extra-pair paternity, and to lower information content than the GRM. Genetic correlations among traits were similar between PED-social and GRM but credible intervals were very large in both cases, suggesting a lack of power for this small dataset. Although a positive linear relationship was found between the number of genes per chromosome and the chromosome heritability for tarsus length, chromosome partitioning similarly showed a lack of power for the three other traits. We discuss the usefulness and limitations of the quantitative genetic inferences based on genomic data in small samples from wild populations. 


\section{Introduction}

Estimating additive genetic variance and heritability of quantitative traits is a major aim in evolutionary biology because they are crucial components of all evolutionary models. Further, fine-scale dissection of the genomic basis of quantitative traits in the wild, using methods such as genome wide association and linkage mapping, requires validation that the traits being mapped are actually heritable. New sequencing technologies have empowered the estimation of genomic relatedness matrices (GRM) between individuals (Visscher et al. 2008; Huisman 2017; Gienapp et al. 2017), feasibly without any social pedigree (PED-social) recorded in the field. Since in a quantitative framework, common SNPs additively explain a large part of genetic additive variance, it is possible to use such realized relatedness estimated from genomic data to measure traits' heritability in wild populations (Yang et al. 2010). Furthermore, by aligning markers against a reference genome, heritability can then be partitioned among chromosomes (or at other genomic scales) enabling testing quantitative models of increasing heritability with the number of genes per chromosomes (Yang et al. 2011b), or alternatively, identifying chromosomes displaying higher genetic variance than expected. GRM estimates and chromosome partitioning, first applied on human data (Yang et al. 2010; 2011b), were then applied in well-known free-ranging animal populations (Great tits, Parus major, Robinson et al. 2013; Soay sheep, Ovis aries, Bérénos et al. 2015) for which thousands of individuals were genotyped at thousands of loci. There have been fewer empirical applications of these methods to smaller samples (but see Wenzel et al. 2015; Silva et al. 2017), although they have great potential in the current context of genomic tools 
being increasingly applied to natural populations (Gienapp et al. 2017) that often rely on relatively small samples.

Three important limitations for estimating GRM and GRM based heritability are the number of SNPs, the number of individuals and the sampling variance (Visscher \& Goddard 2015). The limitation from the number (as well as diversity and quality) of markers and their ability to resolve relatedness essentially vanished when we entered the genomic era (Csillery 2006; Stanton-Geddes et al. 2013; Gienapp et al. 2017). The sample size of individuals genotyped and phenotyped, however, remains an issue. For a given dataset, the number of individuals included will influence both error and median values of heritability estimates, as tested in two studies (Stanton-Geddes et al. 2013; Visscher \& Goddard 2015). Nevertheless, reasonable heritability estimates can be reached using 150 to 200 individuals, as showed in a study on barrelclover (Medicago trunculata, Stanton-Geddes et al. 2013). However, different species, demographic contexts, and sampling strategies will likely perform differently for a similar number of individuals, number of markers and traits' architecture. Visscher \& Goddard (2015) showed that for designs that use genetic markers to estimate relatedness among randomly sampled individuals from a population (which may be a common situation for non-model species in natural populations), the error in heritability estimation is inversely proportional to the squared sample size and is proportional to the effective population size. Therefore, heritability estimates based from genetic marker relatedness in extremely large populations will likely require thousands of samples (e.g. marine pelagic fish (Gagnaire \& Gaggiotti 2016)), but also thousands of loci (given the small linkage disequilibrium among loci). Nevertheless, for populations of smaller size, a sampling 
strategy aiming at capturing variance in realized relatedness offers possibilities to obtain robust heritability estimates bounded by reasonable standard errors.

While genomics offers promising potential to obtain quantitative genetic parameters for virtually any species or population (Gienapp et al. 2017), it has only been used in a few studies to-date and more applications in smaller samples and non-model species are needed. The first studies to estimate genomic heritability and partition variation across the genome elsewhere than in humans were, understandably, focusing on already well-known populations and species. In particular, analysis of 2644 individuals at 7203 SNPs from a long term population study of great tits was the first application of quantitative genomics to a wild population (Robinson et al. 2013). The Soay sheep (Ovis aries) also benefited from such a genomic quantitative genetic study, with 5805 individuals genotyped at 37,037 autosomal SNPs (Bérénos et al. 2014). This study revealed that most of the additive genetic (co)variances in sheep body size traits were captured by half of the SNPs. These pioneering studies enabled the verification of the power of quantitative genomics on known populations with long-term pedigrees and characterized quantitative genetic parameters (Edwards 2013). Other authors also estimated heritability from GRM in datasets with much less individuals, e.g. 200 barrelclover (Medicago trunculata) individuals genotyped at more than 5 million SNPs. Wenzel et al. (2015) estimated genome-wide heritability in 695 red grouse (Lagopus lagopus scotica) genotyped at 384 SNPs. Silva et al. (2017) estimated quantitative genomic parameters in 1,898 house sparrows (Passer domesticus) genotyped at 6,348 SNPs and in 825 collared flycatchers (Ficedula albicolis) genotyped at 38,689 SNPs. These examples confirmed the usefulness of the method in new cases and with fewer 
109 individuals. Although we did not perform a thorough meta-analysis, there was a trend 110 among these studies for an increase, among and within studies, of heritability estimates' 111 standard errors with decreasing sample size and SNP amount. Nevertheless, and 112 surprisingly considering the number of studies performing genomic analyses since the 113 beginning of the genomic era, there are relatively few applications of genomic data to 114 estimate heritability, genetic correlations and chromosome partitioning, using smaller 115 datasets.

116 Here, we assessed heritability, genetic correlations, and chromosome partitioning 117 for four phenotypic traits, namely tarsus length, wing length, bill length, and body mass, 118 in a collection of 494 individuals of Corsican blue tits (Cyanistes caeruleus ogliastrae) 119 from three closely located sites in Corsica (Figure 1). Based on mitochondrial genetic 120 and phenotypic data, blue tits in Corsica and Sardinia have been qualified as a 121 subspecies, Cyanistes caeruleus ogliastrae (Kvist et al. 2004). The focal populations are 122 ideal for quantitative genetic study because of the availability of pedigree, phenotypic 123 and genetic data gathered through a long-term study (Charmantier et al. 2016). Based 124 on microsatellite and SNP genetic data, important gene flow together with significantly 125 low genetic differentiation have been suggested between Corsican populations (Porlier 126 et al. 2012; Szulkin et al. 2016). Phenotypic variance for the four aforementioned traits 127 has been characterized as quantitative and moderately to highly heritable (Charmantier 128 et al. 2004b; Blondel et al. 2006; Charmantier et al. 2016). The latest published results 129 based on 17 to 27 years of monitoring, provided population-specific significant 130 heritability ranging from 0.43 to 0.57 for tarsus length, $0.20-0.33$ for wing length, $0.18-$ 131 0.34 for bill length, and $0.22-0.32$ for body mass. All trait combinations showed 
132 significant additive genetic covariance $\left(\mathrm{COV}_{\mathrm{A}}\right)$ in at least one population; in particular 133 COVAS between wing length and tarsus length and between wing length and body mass 134 were significantly positive (Delahaie et al 2017). Lastly, from 18 to $25 \%$ of young have been identified as extra-pair offspring in these Corsican blue tit populations (Charmantier

et al. 2004a), which may lead to slight underestimation of trait heritability from a fieldbased social pedigree (Charmantier \& Réale 2005).

The general objective of this study was to test the effectiveness of GRM to estimate heritability and genetic correlations among traits and to perform heritability partitioning while using a relatively small sample size of Corsican blue tits. We produced a dataset of 494 Corsican blue tits, from three sites (sample sizes of 110, 185 and 199), genotyped at nearly 50,000 filtered SNPs derived from RAD-seq and phenotyped for four quantitative phenotypic traits (tarsus length, wing length, bill length, and body mass). We looked for erroneous links in the social pedigree (PED-social) using the genetic data, in order to build a corrected social pedigree (PED-corrected). We estimated heritability using both GCTA (Yang et al. 2011a) and MCMCgImm (Hadfield 2010) for the 494 genotyped individuals (with the three sites pooled or separately), based on GRM, PED-social and PED-corrected. Heritability differences between PEDcorrected and PED-social were computed in order to infer the possible effects of pedigree errors on estimates of traits heritability (notably underestimation originating from extra-pair copulations (Charmantier \& Réale 2005)). Comparisons between heritability estimated using PED-corrected and GRM aimed at defining the effect of the greater precision and information content of the GRM. We estimated the effect of the number of SNPs on tarsus length heritability estimates, in order to discuss the SNP 
155 density needed to recover much of the additive genetic variance. Finally, we estimated 156 genetic correlations between traits and we partitioned heritability between

157 chromosomes, although these quantitative genomic tests may challenge our sample 158 size and probably require much larger sample size to produce accurate results. We 159 discuss the usefulness and limitations of these heritability inferences in small samples 160 from wild populations of non-model species.

\section{Methods}

163 Study sites, bird monitoring and phenotypic data

164 Data were collected in three locations (E-PIRIO, D-MURO and E-MURO) in Corsica 165 (France, Figure 1, Table 1). Approximately six kilometers separate D-MURO from EMURO and $27 \mathrm{~km}$ separate these sites from E-PIRIO. The landscape of these sites is 167 dominated either by the evergreen holm oak Quercus ilex (E-PIRIO and E-MURO) or by 168 the deciduous downy oak Q. pubescens (D-MURO). All sites were monitored as part of 169 a long-term research programme for 20 (E-Muro), 24 (D-Muro) and 42 years (E-Pirio) until 2017. A total of 360 nest-boxes were monitored and 26,650 birds were ringed.

171 Capture and handling of the birds was conducted under permits provided by the Centre 172 de Recherches sur la Biologie des Populations d'Oiseaux (CRBPO) and by the Direction 173 Départementale des Services Vétérinaires (DDSV).

174 Each year, nest boxes were visited at least once a week during the reproductive 175 period (from early April to late June). Breeding blue tits were captured in nest boxes 176 during the feeding of their young, and banded (if not already banded earlier) with a 
177 unique metal ring provided by the CRBPO. Nestlings were also banded before fledging 178 at $9-15$ days old.

Four phenotypic traits were measured on male and female breeders: tarsus 180 length (from the intertarsal joint to the most distal undivided scute on the tarsometarsus), flattened wing length, bill length (from the anterior end of the nares to the tip of the upper mandible), and body mass. Phenotypic differences among individuals from the different sites and used in genomic heritability estimates are presented in Table 1 and Supplementary material 1 (see (Charmantier et al. 2016) for a review of phenotypic divergence in these sites). Five to $20 \mu \mathrm{l}$ of blood were sampled from a small neck vein or from a wing vein from adult breeders for later DNA extraction. Blood was stored at $4^{\circ} \mathrm{C}$ 187 in Queen's buffer (Seutin et al. 1991).

DNA extraction, RAD-sequencing, SNP calling and data filtering

We selected a set of 494 individuals captured between the years 2010-2016, from which we extracted DNA from collected blood. The individuals were captured from three sites (E-PIRIO, n = 185; D-MURO, $n=199 ;$ E-MURO, $n=110 ;$ Table 1). Individuals were chosen according to the presence of several phenotypic measurements and a blood sample. DNA extraction was achieved using Qiagen DNeasy Blood \& Tissue kits. DNA extractions were randomized across sites. DNA was quantified using first a NanoDrop ND8000 spectrophotometer and then a Qubit 2.0 fluorometer with the DNA HS assay kit (Life Technologies). DNA quality was checked on agarose gels.

Library preparation using restriction-site-associated DNA sequencing (RAD-seq; Baird et al. 2008) with the enzyme Sbfl was done by MGX (CNRS, Montpellier). Each 
200 individual was identified using a unique 6 nucleotide tag and individuals were 201 multiplexed in equimolar proportions by groups of 36 individuals. Each library was 202 sequenced on one of 20 lanes of an Illumina HiSeq 2000 (libraries also included blue tit 203 individuals from another site that was not analyzed in this study).

Raw sequences were inspected with FastQC (Andrews 2010) for quality controls.

205 Potential fragments of Illumina adapters were trimmed with Cutadapt (Martin 2011), 206 allowing for a $10 \%$ mismatch in the adapter sequence. Reads were filtered for overall 207 quality, demultiplexed and trimmed to $85 \mathrm{bp}$ using process_radtags, from the Stacks 208 software pipeline V1.39 (Catchen et al. 2013), , allowing for one mismatch in the produce sam files using default options. Samtools 0.1.19 (Li et al. 2009) was used to 212 build and sort bam files. Back in Stacks V1.39, we used pstacks to treat bam files, align 213 the reads into matching stacks, infer loci, and detect SNPs at each locus. We used a 214 minimum depth of coverage $(\mathrm{m})$ of 5 , the SNP model, and alpha $=0.05$ (chi square 215 significance level required to call a heterozygote or homozygote). cstacks was used to 216 build the catalogue of loci using $n=3$ (number of mismatches allowed between sample 217 loci when build the catalog). sstacks was used to match loci against the catalog. 218 populations, the last Stacks program used here, genotyped individuals. Loci were 219 retained if genotyped in at least $90 \%$ of individuals (all individuals from all sites 220 grouped), with heterozygosity per site $\leq 0.60$, and with individual minimal read depth of 22110 ("na" replaced genotypes below a read depth of 10). VCFtools (Danecek et al. 2011) was used for further filtering of loci for a minimum average read depth of 20 across all 
223 genotypes, and a maximum average read depth of 100 across all genotypes. Individuals 224 were genotyped for at least $90 \%$ of all loci; a filter would have otherwise been 225 implemented to remove individuals with low genotyping rate. The dataset was filtered to 226 retain loci with minimum allele frequency larger than $5 \%$ (MAF $\geq 0.05$ ). Subsequently the 227 dataset was pruned for linkage disequilibrium with the plink command LD indep 5052.

229 Population genetic structure and genome wide relatedness matrix

230 Data were converted to several formats using the $R$ package radiator (Gosselin 2017).

231 Expected heterozygosity per site and Fst between sites were estimated using Genodive 232 (Meirmans \& van Tienderen 2004). A PCA resolving genetic distance between 233 individuals was calculated using the R packages gdsfmt and SNPRelate (Zheng et al. 234 2012).

235 Genome wide relatedness matrices (GRM) were computed with GCTA (Yang et 236 al. 2010; 2011a) using the markers found on autosomes (i.e. excluding markers from 237 sex chromosomes). We estimated GRMs for each of the three sites and for the three 238 sites pooled. The GRM based on the three sites pooled was represented using a 239 heatmap and histograms in order to depict relatedness diversity in the different sites 240 (Figure 2). Finally, we computed several other GRMs for the unique purpose of 241 chromosome partitioning (detailed in the corresponding section).

243 Social pedigree

244 Social pedigrees (PED-social) were constructed using the pedantics R-package 245 (Morrissey \& Wilson 2009), on the basis of the complete pedigree generated from the 
246 whole long-term monitoring period. We first included all ringed individuals and assigned 247 their mother and father based on observational data at capture. Unknown parents of a

248 given nest were coded using a dummy identity in the PED-social to preserve sibship 249 information. PED-social were pruned for each dataset studied (each population 250 separately, and the combined pedigree of the three sites pooled) to retain only 251 genotyped individuals and their ancestors (see supplementary material 2 for detailed 252 characteristics of the pedigree).

Comparison between social pedigree and genome wide relatedness matrix and creation of a corrected social pedigree

256 We compared the PED-social and GRM for the 494 genotyped individuals mainly in order to infer the potential presence of erroneous pedigree relationship (particularly 258 parent-offspring and sibling relationship). Indeed, errors may occur in social pedigrees, essentially due to extra-pair paternities, but also observational errors such as misreading of bird ring, successive egg-laying by different females in the same nest box, and brood parasitism. Such errors, notably extra-pair paternities, can downwardly bias estimates of heritability (Charmantier \& Réale 2005). We illustrated the concordances and 263 discrepancies between PED-social and GRM using a biplot (Figure 2D). We then created corrected social pedigrees (PED-corrected) on the basis of discrepancies found between the PED-social and the GRM. For pairs of individuals having PED-social relatedness values of 0.5 (full-sibs or parent-offspring relationships) but GRM relatedness estimates below 0.1 , we assumed that these relationships corresponded to erroneous parent-offspring relationship due to extra-pair paternity and assigned them a 
2690 in PED-corrected. For PED-social relatedness values of 0.5 but GRM estimates 270 between 0.15 and 0.35 , we assumed they corresponded to full-sibs being half-sibs and 271 assigned them a value of 0.25 in PED-corrected. We also corrected false half-sibs in the 272 pedigree (pairs of individuals having a 0.25 relatedness value in the PED-social but a

273 GRM estimates smaller than 0.1 ) and assigned them a 0 relatedness values. We 274 acknowledge that probably some more relationships could have been corrected based 275 on discrepancies between PED-social and GRM, but the distribution of GRM values 276 below 0.15 were largely overlapping and it would have been difficult to assign correct 277 PED-corrected values. Using a method such as the one implemented in the R package 278 sequoia (Huisman 2017) would certainly have allowed the correction of these potential errors as well. Another potential limitation comes from the fact that ancestors of the genotyped individuals in the pedigree were not genotyped and therefore erroneous 281 relationships between these individuals were impossible to correct.

Inference of heritability

284 We used both a frequentist and a Bayesian method to estimate heritability of the four traits. The frequentist method was implemented in GCTA. The Bayesian framework was 286 implemented in the MCMCgImm R-package (Hadfield 2010). Bayesian inference is renowned to have a clear advantage over the other existing methods since the use of 288 posterior distributions propagates the errors in estimates derived from animal models 289 (Morrissey et al., 2014).

GCTA was used to estimate heritability for each trait, separately for each of the 291 four GRMs. We here used best linear unbiased estimates (BLUPs) for each individual 
292 and phenotype since we could not implement more complex models in GCTA. BLUPs 293 were estimated for the entire dataset of 494 genotyped individuals using a generalized 294 linear mixed model with the MCMCgImm function, integrating a random effect for the 295 observer and permanent environment effects accounting for multiple measurements of 296 the same individual. Differences between sites and sex were also accounted for by 297 adding these two factors as fixed effects. We then extracted the posterior mode of the 298 BLUPS for each individual, and ran GCTA for each of the four GRMs and the four traits. MCMCgImm was used to estimate heritability for each trait, separately for each of the four GRMs, the four PED-social and the four PED-corrected. Sex was integrated as a fixed effect in all models. Site was integrated as a fixed effect in the models that 302 included all the individuals from the different sites. Measurer, identity and animal (corresponding either to the GRM, PED-social or PED-corrected matrix) effects were 304 incorporated as random factors in order to partition the phenotypic variance into its observer, permanent environment and additive genetic components. Running models based on the GRMs, we used both BLUPs (to compare with GCTA) and alternatively all phenotypic measurements. For the models using GRMs and BLUPs, the only random factor was the animal effect (ie. the GRM) as permanent environmental and observer effects were already taken into account in the BLUPs. For the models based on PEDsocial and PED-corrected, we did not use BLUPs (since it could bias the analysis and/or 311 give anticonservative results, Hadfield et al. 2010) but instead used all phenotypic 312 measurements. We used identical parameters, priors and iterations for each estimate. 313 Random effects included additive genetic effects $\left(V_{A}\right)$ estimated through the inclusion of 314 pedigree data, permanent environmental effects $\left(V_{\mathrm{PE}}\right)$ accounting for repeated 
315 measurements of the same individual, measurer identity controlling for any potential 316 confounding measurer effect $\left(V_{O B S}\right)$ and residual variance $\left(V_{R}\right)$. The models used can be 317 described as follow:

$$
\mathrm{Y}=\mu+X b+Z_{A} a+Z_{P E} p e+Z_{O} o b s+e \text { Equation } 1
$$

319 Equation 1 describes the animal models run on phenotypic traits with $\mathrm{Y}$ the vector of 320 phenotypic observations for all individuals and $\mu$ the vector of mean phenotypes. $\mathrm{Xb}$ 321 stands for the fixed effects (containing sex and site for the models on all individuals, and 322 sex only for within site models). $Z_{A}, Z_{P E}$ and $Z_{O}$ correspond to the random factors:

323 additive genetic (a), permanent environment (pe), and measurer (obs) random effects, 324 respectively. e is the vector of residual errors. Posterior distributions were composed of 3251,000 values per parameter. We used 120,000 iterations per model with sampling every 326100 steps and with 20,000 discarded burn-in iterations. We used slightly informative 327 priors to facilitate convergence, with $V=V_{P} /(r+1), n u=1, V_{P}$ being the phenotypic 328 variance, and $r$ the number of random factors (results were quantitatively and 329 qualitatively similar using uninformative priors, $V=1, n u=0.002$, but convergence takes 330 a bit longer). We checked the models graphically. We verified that autocorrelations were 331 less than 0.05 . We finally reported posterior median, posterior mode, and $95 \%$ credible 332 interval.

Inference of the effect of the number of SNPs on heritability estimates

335 We inspected whether the number of SNPs included resulted in i) an increase in 336 heritability estimation precision (approximated via standard deviation, and LRT 
337 (Likelihood Ratio Test) in the case of GCTA) and ii) an increase in heritability (median) 338 via saturation of the GRMs by SNPs in linkage disequilibrium with loci most likely 339 causative of the phenotypic variation (Gienapp et al. 2017). We reported the median and 340 standard deviation values of tarsus length heritability inferred using GCTA and 341 MCMCgImm for several GRMs produced using a variable number of SNPs. Using 342 GCTA, we analyzed 991 GRMs made up of a decreasing number of randomly chosen 343 SNPs from the entire dataset, by step of 50 SNPs from the total number of SNPs. To 344 confirm GCTA results, we analyzed a smaller number of 25 GRMs with MCMCgImm 345 (much less than for GCTA since the Bayesian analysis was much more time consuming 346 than the frequentist one) made from randomly chosen SNPs, concentrated mainly 347 between 1000 to 10,000 SNPs (since GCTA indicated that the rate of improvement in 348 estimates was particularly concentrated between these numbers).

350 Inference of genetic correlations

351 We used bivariate models in GCTA and in MCMCgImm in order to estimate genetic 352 correlations between each of the four traits. These bivariate models were achieved using 353 the same data, same fixed and random effects, and same number of iterations in the 354 case of $M C M C g / m m$, as for the univariate models. For $M C M C g / m m$, we used slightly

355 informative priors with $V=\left[\begin{array}{cc}\frac{V_{P}}{r} & 0 \\ 0 & \frac{V_{P}}{r}\end{array}\right]$ and $n u=2$ with $V p$ being the phenotypic variance, 356 and $r$ the number of random factors. 


\section{Chromosome partitioning}

Finally, we partitioned heritability across the chromosomes using GCTA, using two methods: i) fitting the univariate GCTA model simultaneously on each GRM corresponding to each autosome; ii) fitting $\mathrm{n}$ times, where $\mathrm{n}$ is the number of autosomes, the univariate GCTA model simultaneously on two GRMs, one GRM computed for the focal chromosome and the other GRM computed using the other autosomes pooled. In both cases (i) and (ii), several microchromosomes (22, 27, 28, 25LG1, 25LG2, LGE22) were pooled into one artificial autosome because they had too few SNPs. When a model did not converge, we discarded the smallest autosomes and ran the model again. We did so until the model would converge.

\section{Results}

\section{Phenotypes}

Among the 494 genotyped individuals, 494, 493, 470, and 488 were measured for tarsus length, wing length, bill length, and body mass, respectively (Table 1, Supplementary material 1). The sex ratio of genotyped individuals was near 0.5 , with 246 females out of 494 individuals. Phenotypic traits varied slightly between sexes and between sites, justifying the inclusion of sex and site effects in the models partitioning phenotypic variance.

\section{SNP calling and population genetic structure}

The median number of reads per individuals was $6,066,514$. The median read depth per individual and per locus was 56 . The Stacks program population outputted 52,783 loci 
381 totaling 96,009 SNPs. After MAF pruning, we retained 41,986 loci totaling 68,114 SNPs.

382 After LD pruning, we retained 38,030 loci totaling 49,682 SNPs. 47,865 of these SNPs were on autosomes. The number of filtered SNPs per chromosome ranged from 5523 384 (chromosome 2) to 56 (LGE22).

The heterozygosity was $0.205,0.205$ and 0.204 in E-PIRIO, D-MURO and EMURO, respectively (Supplementary material 3). Genetic differentiation between sites estimated using an Fst index was low yet significant, ranging from 0.006 to 0.008 (pvalue $<0.001$; Supplementary material 3 ). The two first axes of the PCA (Figure 2A) explaining each $0.98 \%$ and $0.93 \%$ of the genotypic variance, and the heatmap of the GRM (Figure 2B), depicted the low genetic structure between the three sites.

Genome wide relatedness matrix and social pedigree

The GRM (Figure 2C) mostly included non-related (or distantly related) individuals. Zooming in the histogram (Figure 2C) shows the presence of related individuals with parent-offspring or full sib-like links (grey), and half-sib-like links (blue). Comparing the relatedness values from the GRM to the one from the PED-social showed great consistency between the two matrices (Figure 2D). However, as expected (and shown elsewhere, Charmantier and Réale 2005), 112 links out of 858 known links presented PED-social relatedness higher than GRM relatedness by at least 0.10 . Among these, 39 400 links exhibited PED-social relatedness of 0.5 but GRM relatedness of 0 , and 36 links 401 showed PED-social relatedness of 0.25 but GRM relatedness of 0 . In addition, the GRM allowed the identification of many links that cannot be documented in the PED-social 
404 Notably, we found 460 links with PED-social relatedness was equal to 0 but GRM 405 relatedness larger than 0.1 . In total, we corrected 83 erroneous PED-social relatedness 406 values (corresponding to ca. $10 \%$ of the total number of links known) thanks to the GRM 407 analysis to create the PED-corrected.

409 Heritability estimates

410 In general, heritability measures based on PED-social, PED-corrected and GRM, using 411 BLUPs or all the measurements, and with $M C M C$ glmm or GCTA, were highly consistent 412 for the same trait (Table 2, Figure 3; see supplementary material 4 for variance 413 estimates associated with the other random factors). The variations were very high 414 among the three localities, for which sample sizes were very small. When pooling the 415 three localities, variations between methods and credible intervals decreased.

416 Considering estimates from $\mathrm{MCMCg} / \mathrm{mm}$ and models fitting all phenotypic 417 measurements, there was a consistent trend for lower heritability when using PED-social 418 compared to GRM (Figure 3). For example, tarsus length and body mass heritability 419 were lower by $22 \%$ and $31 \%$, respectively, when using PED-social compared GRM. 420 Specifically, there was a trend for lower heritability when using PED-social than PED421 corrected. For example, tarsus length and body mass heritability were lower by $7 \%$ and $42211 \%$, respectively, when using PED-social compared to PED-corrected. There was also 423 a trend for lower heritability when using PED-corrected compared to GRM. For example, 424 tarsus length and body mass heritability were lower by $15 \%$ and $22 \%$, respectively, 425 when using PED-social compared to PED-corrected. 
Heritability estimates were slightly higher when using BLUPs instead of all the 427 phenotypic measures, together with the GRM, in MCMCgImm (e.g. only 3\% higher for 428 tarsus length, but $24 \%$ higher for body mass). Furthermore, credible intervals were 429 slightly higher when using BLUPs. Using BLUPs and the GRM in GCTA, heritability 430 estimates were relatively similar to the one obtained using $M C M C g / m m$ on all the 431 phenotypic measures and either the GRM or the PED-corrected.

432 The number of SNPs included to compute GRMs influenced the tarsus length 433 heritability estimates using either GCTA on BLUPs or MCMCgImm on all of the 434 phenotypic measures (Figure 4). For this trait, heritability sharply increased from a 435 handful of SNPs to approximately 5,000 SNPs. Heritability increased slowly from 436 approximately 5,000 to 15,000 SNPs and then reached a plateau along which a very 437 slow increase was however apparent. It is also worth mentioning a similarly shaped 438 increase of likelihood ratio tests from GCTA.

440 Genetic correlations between traits

441 In general, genetic correlations between traits calculated with the different methods, 442 were consistent for the same pair of trait (Table 3). However, the credible intervals were 443 generally large, with 13 of the 30 intervals including 0 . The largest estimates and 444 smallest credible intervals were obtained for the genetic correlation between tarsus 445 length and body mass, the most heritable traits. For each pair of traits there was a trend 446 for a higher genetic correlation when using all measures than BLUPs. 
449 When fitting the univariate GCTA model simultaneously on each autosome, the 450 correlation between heritability and chromosome length was positive for tarsus length ( $\mathrm{r} 2$ $451=0.26 ; p$-value $=0.009)$, being the most heritable traits of this study. The correlation was 452 non-significant for body mass $(r 2=0.08 ; p$-value $=0.20)$, wing length $(r 2=0.03 ; p$-value $453=0.41)$, and bill length $(r 2=0.00 ; p$-value $=0.85)$. Standard error intervals for 454 chromosome scaled heritability did not include zero only for chromosomes $1 \mathrm{~A}$ and 3 for 455 tarsus length (Table 4, Figure 5), and for chromosome 6 for body mass. In the case of 456 tarsus length, no autosome had to be removed to enable the convergence of the model 457 fitting all the autosomes simultaneously. In contrast, for wing length, bill length and body 458 mass, the microchromosomes group had to be removed and respectively one, 12 and 459 three additional autosomes (by order of increasing size) had to be removed to enable 460 convergence of the models. When fitting the univariate GCTA model separately for each 461 autosome versus the rest of the genome, several heritabilities were likely overestimated 462 since the total heritability was larger than with the previous method ( $\mathrm{h} 2=1.47$ vs 0.84 463 for tarsus length, 0.85 vs 0.36 for wing length, 1.11 vs 0.31 for bill length, and 0.95 vs $464 \quad 0.62$ for body mass).

\section{Discussion}

467 Genomic analyses offer great opportunities for measuring relatedness precisely among 468 individuals from natural populations and hence estimating heritability and genetic 469 correlations among traits in the wild (Edwards 2013; Gienapp et al. 2017). Heritability 470 partitioning along the genome can also help in identifying regions explaining the additive 471 genetic variation for polygenic traits (Yang et al. 2011b). Determining trait heritability and 
472 its partitioning along the genome, and understanding trait genetic covariances, are also essential first steps to detect individual loci in the genome that contribute to trait differences between individuals, using for example genome wide association or linkage mapping (Schielzeth \& Husby 2014). Although such approaches have been recently tested in a few datasets of well-studied wild organisms (Robinson et al. 2013; StantonGeddes et al. 2013; Bérénos et al. 2014), we require more explorations on whether smaller datasets could be used for such procedures and on how they compare with the classic animal model approach based on long-term pedigrees. In this context, we used both social pedigrees (PED-social) and genome wide relatedness matrices (GRM), to compute heritability estimates, genetic correlations, and chromosome partitioning for several phenotypic quantitative traits (tarsus length, wing length, bill length, and body mass) in a rather small dataset of individuals sampled in wild populations of Corsican blue tits and genotyped at nearly 50,000 RADseq derived SNPs. We discuss the power of such relatively small GRM, which may be typically produced while studying wild populations of non-model species, to examine the aforementioned quantitative genetic parameters.

Our main findings were i) a high congruence between heritability estimated using GRM, PED-corrected and PED-social. However, the median heritability obtained using the GRM was generally slightly higher than using PED-corrected and PED-social, which may be attributed to absence of erroneous links, greater precision and higher information content of the GRM. ii) The number of markers was not an issue for computing the GRM and estimating heritability, when approximately 15,000 SNPs were reached. iii) Genetic correlations among traits and chromosome partitioning were less 
495 likely than heritability estimates to be very informative or robust given the large credible 496 intervals and the number of non-significant estimates, most likely due to power issues 497 (insufficient number of SNPs per chromosome in the case of the micro-chromosomes 498 and insufficient number of individuals). We thereafter discuss the usefulness and 499 limitations of inferences of quantitative genomic parameters, including heritability, in 500 relatively small sample sizes, with a medium number of genetic markers, for wild 501 populations of non-model species.

Comparing PED-social and GRM

504

The GRM obtained differed in two aspects from the PED-social (Fig 2D): higher density and relatedness variance on one hand, and no pedigree errors on the other hand. The first major difference was that the GRM enabled estimating relatedness among individuals that were not connected in PED-social (Figure 2D; 460 GRM relatedness values were higher than PED-social relatedness values by at least 0.1 ). This concerned individuals at every degree of relatedness but obviously primarily among individuals from disconnected families. Therefore, GRM increased the depth and also the size of the pedigree. This illustrates the usefulness of GRM in cases for which a dense and deep pedigree is difficult to obtain due to for example dispersal, large population size compared to the fieldwork capacity, and family structure (Pemberton 2008).

14 Furthermore, GRM allows capturing much more variance in realized relatedness among 15 individuals, both close and more distantly related (Figure 2D), which may also increase the power of such GRM-based estimates in large and open populations. 
The second major difference between PED-social and GRM was that several 518 individuals had much higher relatedness in PED-social than in the GRM, most likely 519 indicating errors in PED-social. The most common cases consisted of wrongly assigned parentages (relatedness of 0.5 in PED-social and 0 in GRM), half sibs most likely

521 originating from extra-pair paternities (relatedness of 0.5 in PED-social and 0.25 in

522 GRM), and the spread of such errors in the pedigree (other smaller relatedness in GRM 523 than in PED-social). Using this comparison between GRM and PED-social, we created a 524 PED-corrected in which these aforementioned erroneous links, accounting for approximately $10 \%$ of the PED-social links, were corrected. This proportion of erroneous linked corrected in our dataset should be expected since 18 to $25 \%$ of young have been 527 previously identified as extra-pair offspring in these Corsican blue tit populations 528 (Charmantier et al. 2004a).

Heritability estimates from PED-social, PED-corrected and GRM

Heritability estimated for tarsus length ( 0.62 to 0.81 depending on the method, for three 532 sites pooled, see Table 2$)$ and body mass (0.34-0.61) were on the upper limit compared to previous estimates in this species and for other close species (Jensen et al. 2008;

534 Husby et al. 2011; Postma 2014; Delahaie et al. 2017) while heritability for wing length 535 (0.26-0.34) and bill length (0.14-0.21) were rather on the lower ranges usually found. 536 Here again it should be noted that we did not optimize the animal models by integrating 537 factors that may have contributed to explain trait variation, for example to account for 538 daily and seasonal variation in body mass. Moreover, it has been observed that the 539 number of individuals included can affect the heritability estimates (Silva et al. 2017). 
Credible intervals and standard errors for heritability estimates were much larger 541 than in previous studies based on larger sample size (Robinson et al. 2013; Stanton542 Geddes et al. 2013; Bérénos et al. 2014; Silva et al. 2017). The credible intervals per 543 site were larger than for the three sites pooled, suggesting the decrease of power with 544 decreasing sample size. Similarly, Silva et al. (2017) obtained increased standard errors 545 when decreasing sample size. This most likely suggests that the number of individuals used here conferred limited power.

Overall, when using MCMCgImm and models using all of the phenotypic measurements, heritability estimates were relatively similar for GRM, PED-corrected and PED-social for the same trait (Figure 3). These results are in line with previous comparisons realized with much larger sample size (Robinson et al. 2013; Bérénos et al. 2014). However, unlike these previous studies showing very small differences in heritability based on PED-social and GRM, our estimates based on GRM were larger than using PED-social and to a lesser extent, than using PED-corrected, for tarsus length and for body mass, but not for wing length and bill length. Here, wing length heritability was 0.28 using PED-social, 0.26 using PED-corrected and 0.26 using GRM (Table 2). In turn, tarsus length heritability obtained from GRM was $18 \%$ higher than from PED-corrected and the one from PED-corrected 8\% higher than from PED-social. Similarly, body mass heritability obtained from GRM was $29 \%$ higher than from PEDcorrected and the one from PED-corrected 12\% higher than from PED-social. The difference between PED-corrected and PED-social for these two traits may be attributable to the erroneous links in PED-social. Indeed, it has been estimated that PED-social containing 5 to $20 \%$ of extra-pair paternities result in underestimated 
563 heritability by up to $17 \%$, in blue tits (Charmantier \& Réale 2005). Then, the differences 564 observed between heritability estimated using GRM and PED-corrected are likely to 565 originate from higher density of the GRM and the fact that GRM incorporate more 566 variance in relatedness for a given class of kinship. Further changing the pedigree by 567 not only correcting erroneous existing links but also informing previously unidentified 568 links (eg. linking previously unknown extra-pair fathers to their offspring) based on the 569 genetic data, could also decrease the heritability difference between PED-corrected and 570 GRM.

Heritability estimates, and their credible intervals, based on BLUPs were slightly

572 higher compared to estimates based on models using all phenotypic measurements 573 when using MCMCgImm (this was not true for GCTA). This is not surprising given that 574 BLUPs are known to artificially increase the precision of the measure and therefore 575 increase statistical significance (Hadfield et al. 2010). While the difference was low for 576 tarsus and bill length ( $3 \%$ and $5 \%$ higher, respectively), the difference was particularly 577 pronounced for wing length and body mass (31\% and $24 \%$, respectively). One 578 variance.

Effect of the number of SNPs on the heritability estimates based on GRM

583 The number of SNPs had a relatively minor effect on heritability estimates, from 584 approximately 15,000 SNPs (Figure 4). This is concordant with what has been shown in 585 previous studies (Stanton-Geddes et al. 2013; Bérénos et al. 2014). Of course, the effect 
586 of the number of SNPs on the heritability estimation depends on several parameters 587 (e.g. genome size, LD across the genome, LD among markers, genetic architecture of 588 the focal trait, polymorphism of the markers, genotyping quality, missing data) and is not 589 transposable from one study to another. These days, the number of markers may no 590 longer be a problem, given the rise of the accessibility of many genomic tools. However, 591 the genotyping quality and missing data occurrence may be challenging for studies 592 using RADseq and GBS methods, as noted by (Gienapp et al. 2017). Here, we 593 prioritized the quantity and the quality of the RAD markers over the number of 594 individuals, as well as genotyping only individuals with high quality DNA, ending up with 595 a relatively high read depth and low missing rate. Future studies aiming to use RADseq 596 derived markers to estimate heritability should also find a tradeoff between the number 597 of genomic markers and the number of genotyped individuals. A formal simulation study 598 determining such a tradeoff between numbers of individuals and of markers and markers read-depth (directly linked to quality) would be highly valuable for guiding the design of 600 RADseq analyses in the context of quantitative genomics.

603 Genetic correlations obtained using GRM, PED-social and PED-corrected had large 604 credible intervals, preventing us to make robust interpretations, except that our sample 605 size was probably too small to obtain acceptable credible intervals. This was partly 606 expected since bivariate models are known to be data hungry. While such lack of power 607 is a recurrent issue when estimating genetic correlations even with much larger sample 608 sizes (Vattikuti et al. 2012; Visscher et al. 2014; Ni et al. 2017), it was nevertheless a 
609 disappointment while comparing these results to the one from (Bérénos et al. 2014), in 610 which the credible intervals were relatively small. The median values of genetic 611 correlations for these traits were in line with previous studies in other bird species 612 (Teplitsky et al. 2014).

613

614 Chromosome partitioning based on GRM

615 Chromosome partitioning of heritability of tarsus length was on par with what has been 616 observed in previous studies, i.e. increasing heritability of quantitative traits with 617 increasing number of genes per chromosome (Yang et al. 2011b; Robinson et al. 2013; 618 Santure et al. 2013, 2015; Bérénos et al. 2015; Wenzel et al. 2015; Silva et al. 2017). 619 Similarly to several of these previous studies, there were few chromosomes for which 620 standard error did not include zero. Of particular interest, chromosomes 3 and $1 \mathrm{~A}$ 621 showed high heritability for tarsus length. These chromosomes may be of particular 622 interest for future studies investigating the genomic bases of this quantitative trait 623 variation in blue tits. Chromosome 3 was already identified in a recent study on 624 morphology of Dutch and British great tits (Santure et al. 2015) as explaining high 625 heritability for tarsus length. In contrast, chromosome 1A explained a low proportion of 626 genetic variance in this last study. These kind of discrepancies might be congruent with 627 the fact that this same study (Santure et al. 2015) also showed very little consistency 628 between the variance explained by each chromosome for great tit populations from the 629 Netherlands and from the United-Kingdom.

Regarding the three other traits analyzed, the chromosome partitioning results 631 appeared much less robust since several chromosomes had to be removed $(7,18$, and 
6329 autosomes removed, for wing length, bill length and body mass, respectively) to 633 enable models' convergence. The need for such chromosomes removals has previously 634 been reported (Wenzel et al. 2015; Silva et al. 2017) and has received detailed 635 consideration by Kemppainen \& Husby (2018). In addition, the relationship between the 636 number of genes and the heritability explained by chromosomes were deviating from 637 theoretical expectations of increasing heritability with increasing chromosome size for 638 these polygenic traits. For comparison, Bosse et al. (2017) showed a significant increase 639 of the proportion of additive genetic variance of bill length explained by a chromosome in 640 relation to chromosome size in great tits. Such deviation may not have been affected by 641 removing chromosomes (Kemppainen \& Husby 2018) but most likely originated from a 642 limited power of the small sample size, alongside relatively small heritabilities 643 (Kemppainen \& Husby 2018). Conclusion on the usefulness of GRM based heritability measures using small samples from wild populations

647 Overall, our study reveals that RADseq data on around 50k SNPs (as advised by 648 Berenos et al 2014) for around 500 phenotyped individuals can provide estimates of 649 heritability that are close to, and probably more accurate than, estimates based on social 650 pedigree data from seven years monitoring for more than 1600 individuals (as included 651 in the PED-social used here). This opens very interesting avenues in the field of 652 quantitative genetics since estimating heritability and genetic correlations in the wild 653 have long been restricted to study systems where long-term monitoring is feasible. 654 RADseq data could allow the estimation of quantitative genetic parameters for a much 
655 lower cost in terms of time (years) spent in the field, although individual capture and 656 phenotyping will most likely remain time consuming. Using GRM on few individuals gives 657 the power to estimate such parameters on virtually any species even though no long658 term pedigree is available (Gienapp 2017). This will notably allow moving on from 659 individual study to more comparative approaches and answer important questions such 660 as understanding the spatial variation of evolutionary potential or the role of evolutionary 661 constraints in phenotypic evolution. Such a genomic approach also provides the 662 possibility to explore genetic covariance between traits as well as chromosome 663 partitioning to confirm the polygenic architecture for phenotypic traits classically 664 measured in birds. Less than 500 individuals appears however to provide insufficient 665 power to correctly estimate either genetic covariances between traits or contribution of 666 individual chromosomes to overall heritability. 


\section{References}

668

Andrews S (2010) FastQC: A quality control tool for high throughput sequence data. Available online at: http://www.bioinformatics.babraham.ac.uk/projects/fastqc. Baird NA, Etter PD, Atwood TS et al. (2008) Rapid SNP Discovery and Genetic Mapping Using Sequenced RAD Markers. PLoS ONE 3(10): e3376. https://doi.org/10.1371/journal.pone.0003376.

Bérénos C, Ellis PA, Pilkington JG, Pemberton JM (2014) Estimating quantitative genetic parameters in wild populations: a comparison of pedigree and genomic approaches. Molecular Ecology, 23, 3434-3451.

Bérénos C, Ellis PA, Pilkington JG et al. (2015) Heterogeneity of genetic architecture of body size traits in a free-living population. Molecular Ecology, 24, 1810-1830.

Blondel J, Thomas DW, Charmantier A et al. (2006) A Thirty-Year Study of Phenotypic and Genetic Variation of Blue Tits in Mediterranean Habitat Mosaics. Bioscience, 56, $661-673$.

Bosse, M., Spurgin, L. G., Laine, V. N., Cole, E. F., Firth, J. A., Gienapp, P., ... \& Groenen, M. A. (2017). Recent natural selection causes adaptive evolution of an avian polygenic trait. Science, $358,365-368$.

Catchen J, Hohenlohe PA, Bassham S, Amores A, Cresko WA (2013) Stacks: an analysis tool set for population genomics. Molecular Ecology, 22, 3124-3140.

Charmantier A, Réale D (2005) How do misassigned paternities affect the estimation of heritability in the wild? Molecular Ecology, 14, 2839-2850.

Charmantier A, Blondel J, Perret P, Lambrechts M (2004a) Do extra-pair paternities 
provide genetic benefits for female blue tits Parus caeruleus? Journal of Avian Biology, 35, 1-9.

Charmantier A, Doutrelant C, Dubuc-Messier G, Fargevieille A, Szulkin M (2016) Mediterranean blue tits as a case study of local adaptation. Evolutionary Applications, 9, 135-152.

Charmantier A, Kruuk LEB, Blondel J, Lambrechts MM (2004b) Testing for microevolution in body size in three blue tit populations. Journal of Evolutionary Biology, 17, 732-743.

Csillery K (2006) Performance of Marker-Based Relatedness Estimators in Natural Populations of Outbred Vertebrates. Genetics, 173, 2091-2101.

Danecek P, Auton A, Abecasis G et al. (2011) The variant call format and VCFtools. Bioinformatics, 27, 2156-2158.

Delahaie, B., Charmantier, A., Chantepie, S., Garant, D., Porlier, M., \& Teplitsky, C. (2017). Conserved G-matrices of morphological and life-history traits among continental and island blue tit populations. Heredity, 119, 76-87.

Edwards SV (2013) Next-generation QTL mapping: crowdsourcing SNPs, without pedigrees. Molecular Ecology, 22, 3885-3887.

Gagnaire, P. A., \& Gaggiotti, O. E. (2016). Detecting polygenic selection in marine populations by combining population genomics and quantitative genetics approaches. Current Zoology, 62, 603-616.

Gienapp, P., Fior, S., Guillaume, F., Lasky, J. R., Sork, V. L., \& Csilléry, K. (2017). Genomic quantitative genetics to study evolution in the wild. Trends in ecology \& 
evolution, 32, 897-908.

Gosselin T (2017). Radiator: RADseq Data Exploration, Manipulation and Visualization using R. doi: 10.5281/zenodo.154432.

Hadfield JD, Wilson AJ, Garant D, Sheldon BC, Kruuk LEB (2010) The Misuse of BLUP in Ecology and Evolution. The American Naturalist, 175, 116-125.

Hadfield, J. D. (2010). MCMC methods for multi-response generalized linear mixed models: the MCMCgImm R package. Journal of Statistical Software, 33, 1-22.

Huisman J (2017) Pedigree reconstruction from SNP data: parentage assignment, sibship clustering and beyond. Molecular Ecology Resources, 17, 1009-1024.

Husby A, Hille SM, Visser ME (2011) Testing Mechanisms of Bergmann's Rule: Phenotypic Decline but No Genetic Change in Body Size in Three Passerine Bird Populations. American Naturalist, 178, 202-213.

Jensen H, Steinsland I, Ringsby TH, Sæther B-E (2008) Evolutionary dynamics of a sexual ornament in the house sparrow (Passer domesticus): the role of indirect selection within and between sexes. Evolution, 62, 1275-1293.

Kemppainen, P., \& Husby, A. (2018). Inference of genetic architecture from chromosome partitioning analyses is sensitive to genome variation, sample size, heritability and effect size distribution. Molecular Ecology Resources. https://doi.org/10.1111/1755-0998.12774.

Kvist L, Viiri K, Dias PC, Rytkönen S, Orell M (2004) Glacial history and colonization of Europe by the blue tit Parus caeruleus. Journal of Avian Biology, 35, 352-359.

Laine VN, Gossmann TI, Schachtschneider KM et al. (2016) Evolutionary signals of selection on cognition from the great tit genome and methylome. Nature 
Communications, 7, 1-9.

Li H, Durbin R (2009) Fast and accurate short read alignment with Burrows-Wheeler transform. Bioinformatics, 25, 1754-1760.

Li H, Handsaker B, Wysoker A et al. (2009) The Sequence Alignment/Map format and SAMtools. Bioinformatics, 25, 2078-2079.

Martin M (2011) Cutadapt removes adapter sequences from high-throughput sequencing reads. EMBnet, 17, 10-12.

Meirmans PG, van Tienderen PH (2004) genotype and genodive: two programs for the analysis of genetic diversity of asexual organisms. Molecular Ecology Notes, 4, 792794.

Morrissey, M. B., de Villemereuil, P., Doligez, B., \& Gimenez, O.. (2014). Bayesian approaches to the quantitative genetic analysis of natural populations. In: A. Charmantier, D. Garant, and L. E. B. Kruuk, eds. Quantitative Genetics in the Wild, pp. 228-253. Oxford University Press, Oxford, UK.

Morrissey MB, Wilson AJ (2009) pedantics: an r package for pedigree-based genetic simulation and pedigree manipulation, characterization and viewing. Molecular Ecology Resources, 10, 711-719.

Ni, G., Moser, G., Wray, N. R., \& Lee, S. H. (2017). Estimation of genetic correlation using linkage disequilibrium score regression and genomic restricted maximum likelihood. bioRxiv, 194019.

Pemberton JM (2008) Wild pedigrees: the way forward. Proceedings of the Royal Society B-Biological Sciences, 275, 613-621.

Porlier, M., Garant, D., Perret, P., \& Charmantier, A. (2012). Habitat-linked population 
genetic differentiation in the blue tit Cyanistes caeruleus. Journal of Heredity, 103(6), 781-791.

Postma E (2014) Four decades of estimating heritabilities in wild vertebrate populations: improved methods, more data, better estimates? In: A. Charmantier, D. Garant, and L. E. B. Kruuk, eds. Quantitative Genetics in the Wild, pp. 16-33. Oxford University Press, Oxford, UK.

Robinson MR, Santure AW, DeCauwer I, Sheldon BC, Slate J (2013) Partitioning of genetic variation across the genome using multimarker methods in a wild bird population. Molecular Ecology, 22, 3963-3980.

Santure, A. W., Poissant, J., De Cauwer, I., Oers, K., Robinson, M. R., Quinn, J. L., ... \& Slate, J. (2015). Replicated analysis of the genetic architecture of quantitative traits in two wild great tit populations. Molecular ecology, 24, 6148-6162.

Santure, A. W., Cauwer, I., Robinson, M. R., Poissant, J., Sheldon, B. C., \& Slate, J. (2013). Genomic dissection of variation in clutch size and egg mass in a wild great tit (Parus major) population. Molecular Ecology, 22, 3949-3962.

Schielzeth, H., \& Husby, A. (2014). Challenges and prospects in genome-wide quantitative trait loci mapping of standing genetic variation in natural populations. Annals of the New York Academy of Sciences, 1320, 35-57.

Seutin G, White BN, Boag PT (1991) Preservation of avian blood and tissue samples for DNA analyses. Canadian Journal of Zoology-Revue Canadienne De Zoologie, 69, 82-90.

Silva, C. N. S., McFarlane, S. E., Hagen, I. J., Rönnegård, L., Billing, A. M., Kvalnes, T., 

... \& Qvarnström, A. (2017). Insights into the genetic architecture of morphological traits in two passerine bird species. Heredity, 119, 197-205.

Stanton-Geddes J, Yoder JB, Briskine R, Young ND, Tiffin P (2013) Estimating heritability using genomic data (J Hadfield, Ed,). Methods in Ecology and Evolution, 4, 1151-1158.

Szulkin M, Gagnaire PA, Bierne N, Charmantier A (2016) Population genomic footprints of fine-scale differentiation between habitats in Mediterranean blue tits. Molecular Ecology, 25, 542-558.

Vattikuti S, Guo J, Chow CC (2012) Heritability and Genetic Correlations Explained by Common SNPs for Metabolic Syndrome Traits (PM Visscher, Ed,). Plos Genetics, 8, e1002637-8.

Visscher PM, Goddard ME (2015) A General Unified Framework to Assess the Sampling Variance of Heritability Estimates Using Pedigree or Marker-Based Relationships. Genetics, 199, 223-232.

Visscher PM, Hemani G, Vinkhuyzen AAE et al. (2014) Statistical Power to Detect Genetic (Co)Variance of Complex Traits Using SNP Data in Unrelated Samples (GS Barsh, Ed,). Plos Genetics, 10, e1004269.

Visscher PM, Hill WG, Wray NR (2008) Heritability in the genomics era - concepts and misconceptions. Nature Reviews Genetics, 9, 255-266.

Wenzel MA, James MC, Douglas A, Piertney SB (2015) Genome-wide association and genome partitioning reveal novel genomic regions underlying variation in gastrointestinal nematode burden in a wild bird. Molecular Ecology, 24, 4175-4192. 
801 Yang J, Benyamin B, McEvoy BP et al. (2010) Common SNPs explain a large proportion 802 of the heritability for human height. Nature Genetics, $42,565-569$.

803 Yang J, Lee SH, Goddard ME, Visscher PM (2011a) GCTA: a tool for genome-wide 804 complex trait analysis. American Journal of Human Genetics, 88, 76-82.

805 Yang J, Manolio TA, Pasquale LR et al. (2011b) Genome partitioning of genetic variation 806 for complex traits using common SNPs. Nature Genetics, 43, 519-525.

807 Zheng X, Levine D, Shen J et al. (2012) A high-performance computing toolset for 808 relatedness and principal component analysis of SNP data. Bioinformatics, 28, $809 \quad 3326-3328$.

810 


\section{Acknowledgments}

812 We thank all the people who helped to maintain the study sites and to conduct the blue

813 tit monitoring, in particular Christophe de Franceschi, Claire Doutrelant, Arnaud 814 Grégoire, Marcel Lambrechts, Jacques Blondel and Philippe Perret. We thank Pierre

815 Alexandre Gagnaire for useful advices on genomics. We thank three anonymous

816 reviewers and Anna Santure for precious comments on earlier versions of this

817 manuscript. This project was funded by the European Research Council (Starting grant

818 ERC-2013-StG-337365-SHE to AC) and a long-term funding from OSU-OREME.

\section{Data archiving}

821 Data (Plink files, PED-social, and repeated and BLUPs phenotypic traits values) are 822 available from the Dryad Digital Repository: doi:10.5061/dryad.k6r1mk8

\section{Conflict of interest}

825 The authors declare no conflict of interest.

\section{Author contributions}

828 Conception of the study: AC \& CP. Field work: AC, BD \& CP. Lab work \& bioinformatics:

829 CP. Quantitative genetic/genomics inferences: CP \& BD. Writing of the manuscript: CP. 830 Critically revised and improved the manuscript: AC, BD \& CP. 


\section{Figure captions}

834 Figure 1. A- Map of the sampling locations in Corsica, including a zoom-in of the area

835 where the populations are located B- D-Muro and E-Muro and C- E-Pirio (see table 1 for 836 details).

838 Figure 2. Genetic structure and relatedness among individuals. A- Biplot of the two first 839 axes of a principal component analysis of genetic distances between individuals, B840 Heatmap of the genome wide relatedness matrix (GRM) among the 494 genotyped 841 individuals, C- histogram of genome wide relatedness (GRM) among individuals, with a 842 zoom-in highlighting particularly high relatedness values, and D- Biplot of genome wide 843 relatedness (GRM) and social pedigree relatedness from the field observations (PED844 social). In B, C and D, color-coding is based on the empirical relatedness distribution 845 and is only indicative.

847 Figure 3. A- Heritability estimates using the GRM, the PED-social or the PED-corrected, based on either BLUPS or the entire phenotypic measurements, and implementing models in GCTA or MCMCgImm, for the 494 genotyped individuals from the three

850 populations pooled. B, C \& D- Relationship between heritability inferred for the four traits 851 for PED-social, PED-corrected and GRM using MCMCgImm, using the model with all 852 phenotypic measurements in MCMCgImm. Errors bars correspond to $95 \%$ credible 853 interval for MCMCgImm and to $95 \%$ confidence intervals for GCTA. 
855 Figure 4. Effect of the number of SNPs used to build the GRM on the tarsus length 856 heritability estimated with GCTA \& BLUPs (A) and MCMCgImm \& all the phenotypic 857 measures (B). Circles represent the median value. Bars represent standard errors for 858 GCTA and $95 \%$ credible interval for MCMCgImm. Black curves correspond to LOESS 859 fits. The log likelihood ratio of GCTA estimates is color-coded.

860

861 Figure 5. Chromosome partitioning. The univariate GCTA model was fitted 862 simultaneously on each autosome. Linear correlation between the number of genes per 863 chromosome and the GCTA estimates of heritability per chromosome for the four 864 phenotypic traits. Names of the largest chromosomes, and of the chromosomes having 865 the highest heritability, are given.

866 


\section{Tables}

868 Table 1. Geographic coordinates, number of genotyped individuals $(\mathrm{N})$, and trait mean values ( \pm SE) for this sample, for 869 each of the three sites and for the entire dataset. The phenotypic variance $(V p)$ is also given for each trait.

\begin{tabular}{|c|c|c|c|c|c|c|c|c|c|c|c|c|c|c|c|c|c|c|c|c|}
\hline \multirow[t]{2}{*}{ Site } & \multirow[t]{2}{*}{ Latitude } & \multirow[t]{2}{*}{ Longitude } & \multirow[t]{2}{*}{$\mathrm{N}$} & \multirow[t]{2}{*}{ Sex } & \multicolumn{4}{|c|}{ Tarsus length (mm) } & \multicolumn{4}{|c|}{ Wing length $(\mathrm{mm})$} & \multicolumn{4}{|c|}{ Bill length $(\mathrm{mm})$} & \multicolumn{4}{|c|}{ Body mass (g) } \\
\hline & & & & & $\mathrm{N}$ & mean & SE & $V p$ & $\mathrm{~N}$ & mean & SE & $V p$ & $\mathrm{~N}$ & mean & SE & $V p$ & $\mathrm{~N}$ & mean & SE & $V p$ \\
\hline \multirow[t]{2}{*}{ E-Pirio } & 42.376 & 8.750 & 185 & male & 98 & 16.4 & 0.5 & & 98 & 63.5 & 1.6 & & 90 & 6.4 & 0.3 & & 97 & 9.4 & 0.4 & \\
\hline & & & & both & 185 & 16.1 & 0.6 & 0.25 & 185 & 62.2 & 2.0 & 4.91 & 172 & 6.5 & 0.4 & 0.16 & 183 & 9.3 & 0.4 & 0.27 \\
\hline \multirow[t]{2}{*}{ D-Muro } & 42.551 & 8.923 & 199 & male & 95 & 16.6 & 0.4 & & 95 & 63.3 & 1.6 & & 92 & 6.4 & 0.3 & & 92 & 9.8 & 0.4 & \\
\hline & & & & both & 199 & 16.3 & 0.5 & 0.24 & 199 & 62.0 & 1.9 & 4.38 & 191 & 6.4 & 0.3 & 0.16 & 196 & 9.6 & 0.5 & 0.27 \\
\hline \multirow[t]{3}{*}{ E-Muro } & 42.589 & 8.967 & 110 & male & 55 & 16.5 & 0.5 & & 55 & 63.4 & 1.5 & & 53 & 6.4 & 0.3 & & 54 & 9.7 & 0.4 & \\
\hline & & & & female & 55 & 16.0 & 0.4 & & 54 & 60.8 & 1.1 & & 54 & 6.7 & 0.5 & & 55 & 9.5 & 0.4 & \\
\hline & & & & both & 110 & 16.3 & 0.5 & 0.25 & 109 & 62.1 & 1.8 & 3.84 & 107 & 6.6 & 0.4 & 0.19 & 109 & 9.6 & 0.4 & 0.24 \\
\hline \multirow[t]{2}{*}{ Total } & & & 494 & male & 248 & 16.5 & 0.4 & & 248 & 63.4 & 1.6 & & 235 & 6.4 & 0.3 & & 243 & 9.6 & 0.4 & \\
\hline & & & & female & 246 & 16.0 & 0.5 & & 245 & 60.7 & 1.2 & & 235 & 6.6 & 0.4 & & 245 & 9.4 & 0.5 & \\
\hline
\end{tabular}


870 Table 2. Heritability estimates (h2) and confidence (GCTA) / credible $(M C M C g / m m)$ intervals $(95 \% \mathrm{Cl})$ for the four traits, 871 using the GRM, the PED-social or the PED-corrected, based on either BLUPS or the entire measures, and implementing models in GCTA or MCMCgImm, for all the individuals or each of the three sites separately. Number of individuals used

$873(\mathrm{~N})$

\begin{tabular}{|c|c|c|c|c|c|c|c|c|c|c|c|c|c|c|c|c|c|c|c|c|}
\hline \multirow{3}{*}{ Relatedness } & \multirow{2}{*}{ Data } & \multirow{3}{*}{ Implementation } & \multirow{2}{*}{ Site } & \multirow{2}{*}{$\mathrm{N}$} & \multicolumn{4}{|c|}{ Tarsus length } & \multicolumn{4}{|c|}{ Wing length } & \multicolumn{4}{|c|}{ Bill length } & \multicolumn{4}{|c|}{ Body mass } \\
\hline & & & & & \multirow{2}{*}{$\frac{N}{489}$} & \multirow{2}{*}{$\frac{\mathrm{h} 2}{0.72}$} & \multicolumn{2}{|c|}{$95 \% \mathrm{Cl}$} & \multirow{2}{*}{$\frac{N}{493}$} & \multirow{2}{*}{$\frac{\mathrm{h} 2}{0.26}$} & \multicolumn{2}{|c|}{$95 \% \mathrm{Cl}$} & \multirow{2}{*}{$\frac{N}{469}$} & \multirow{2}{*}{$\frac{\mathrm{h} 2}{0.16}$} & \multicolumn{2}{|c|}{$95 \% \mathrm{Cl}$} & \multirow{2}{*}{$\frac{N}{488}$} & \multirow{2}{*}{$\frac{\mathrm{h} 2}{0.48}$} & \multicolumn{2}{|c|}{$95 \% \mathrm{Cl}$} \\
\hline & & & 3 pop pooled & 494 & & & 0.5 & 0.94 & & & 0 & 0.48 & & & 0 & 0.4 & & & 0.25 & 0.72 \\
\hline \multirow{3}{*}{ GRM } & \multirow{3}{*}{ BLUPS } & \multirow{3}{*}{ GCTA } & E-Pirio & 185 & 182 & 0.64 & 0.28 & 1 & 185 & 0.25 & 0 & 0.6 & 171 & 0.11 & 0 & 0.46 & 183 & 0.37 & 0 & 0.74 \\
\hline & & & D-Muro & 199 & 197 & 0.73 & 0.38 & 1 & 199 & 0.46 & 0.04 & 0.87 & 191 & 0.22 & 0 & 0.64 & 196 & 0.41 & 0 & 0.88 \\
\hline & & & E-Muro & 110 & 110 & 1 & 0.66 & 1 & 109 & 0.36 & 0 & 0.84 & 107 & 0.59 & 0 & 1 & 109 & 1 & 0.69 & 1 \\
\hline \multirow{4}{*}{ GRM } & \multirow{4}{*}{ BLUPS } & \multirow{4}{*}{ MCMCgImm } & 3 pop pooled & 494 & 489 & 0.81 & 0.63 & 0.92 & 493 & 0.34 & 0.11 & 0.58 & 469 & 0.2 & 0.06 & 0.52 & 488 & 0.61 & 0.38 & 0.83 \\
\hline & & & E-Pirio & 185 & 182 & 0.74 & 0.44 & 0.95 & 185 & 0.31 & 0.08 & 0.66 & 171 & 0.26 & 0.05 & 0.52 & 183 & 0.58 & 0.19 & 0.78 \\
\hline & & & D-Muro & 199 & 197 & 0.8 & 0.42 & 0.96 & 199 & 0.49 & 0.19 & 0.91 & 191 & 0.3 & 0.71 & 0.07 & 196 & 0.58 & 0.11 & 0.86 \\
\hline & & & E-Muro & 110 & 110 & 0.93 & 0.65 & 0.98 & 109 & 0.49 & 0.1 & 0.82 & 107 & 0.35 & 0.11 & 0.95 & 109 & 0.9 & 0.55 & 0.98 \\
\hline \multirow{4}{*}{ GRM } & & & 3 pop pooled & 494 & 489 & 0.79 & 0.65 & 0.87 & 493 & 0.26 & 0.11 & 0.5 & 469 & 0.19 & 0.03 & 0.37 & 488 & 0.49 & 0.33 & 0.64 \\
\hline & II & MCM Calmn & E-Pirio & 185 & 182 & 0.72 & 0.39 & 0.89 & 185 & 0.2 & 0.06 & 0.42 & 171 & 0.13 & 0.02 & 0.35 & 183 & 0.39 & 0.09 & 0.57 \\
\hline & measures & $C M C g I m m$ & D-Muro & 199 & 197 & 0.84 & 0.54 & 0.89 & 199 & 0.48 & 0.18 & 0.73 & 191 & 0.29 & 0.06 & 0.49 & 196 & 0.17 & 0.04 & 0.57 \\
\hline & & & E-Muro & 110 & 110 & 0.86 & 0.61 & 0.91 & 109 & 0.37 & 0.07 & 0.55 & 107 & 0.36 & 0.05 & 0.6 & 109 & 0.66 & 0.42 & 0.78 \\
\hline & & & 3 pop pooled & 494 & 489 & 0.67 & 0.49 & 0.8 & 493 & 0.26 & 0.13 & 0.41 & 469 & 0.21 & 0.06 & 0.3 & 488 & 0.38 & 0.24 & 0.48 \\
\hline PED- & All & $\mathrm{MCMCalmm}$ & E-Pirio & 185 & 182 & 0.54 & 0.22 & 0.78 & 185 & 0.12 & 0.04 & 0.3 & 171 & 0.14 & 0.03 & 0.27 & 183 & 0.25 & 0.07 & 0.41 \\
\hline & & & E-Muro & 110 & 110 & 0.74 & 0.41 & 0.84 & 109 & 0.28 & 0.06 & 0.4 & 107 & 0.18 & 0.05 & 0.43 & 109 & 0.85 & 0.43 & 0.95 \\
\hline & & & 3 pop pooled & 494 & 489 & 0.62 & 0.46 & 0.73 & 493 & 0.28 & 0.13 & 0.37 & 469 & 0.14 & 0.06 & 0.23 & 488 & 0.34 & 0.22 & 0.42 \\
\hline PFD-social & All & MCMCalmm & E-Pirio & 185 & 182 & 0.52 & 0.22 & 0.71 & 185 & 0.12 & 0.03 & 0.26 & 171 & 0.10 & 0.03 & 0.22 & 183 & 0.22 & 0.06 & 0.4 \\
\hline & measure & & D-Muro & 199 & 197 & 0.6 & 0.27 & 0.72 & 199 & 0.37 & 0.16 & 0.52 & 191 & 0.09 & 0.03 & 0.19 & 196 & 0.29 & 0.11 & 0.41 \\
\hline & & & E-Muro & 110 & 110 & 0.68 & 0.38 & 0.78 & 109 & 0.19 & 0.05 & 0.35 & 107 & 0.11 & 0.04 & 0.37 & 109 & 0.4 & 0.17 & 0.53 \\
\hline
\end{tabular}


Table 3. Genetic correlations ( $\mathrm{rG})$, and confidence (GCTA) / credible $(M C M C g / m m)$ intervals $(95 \% \mathrm{Cl})$, between traits

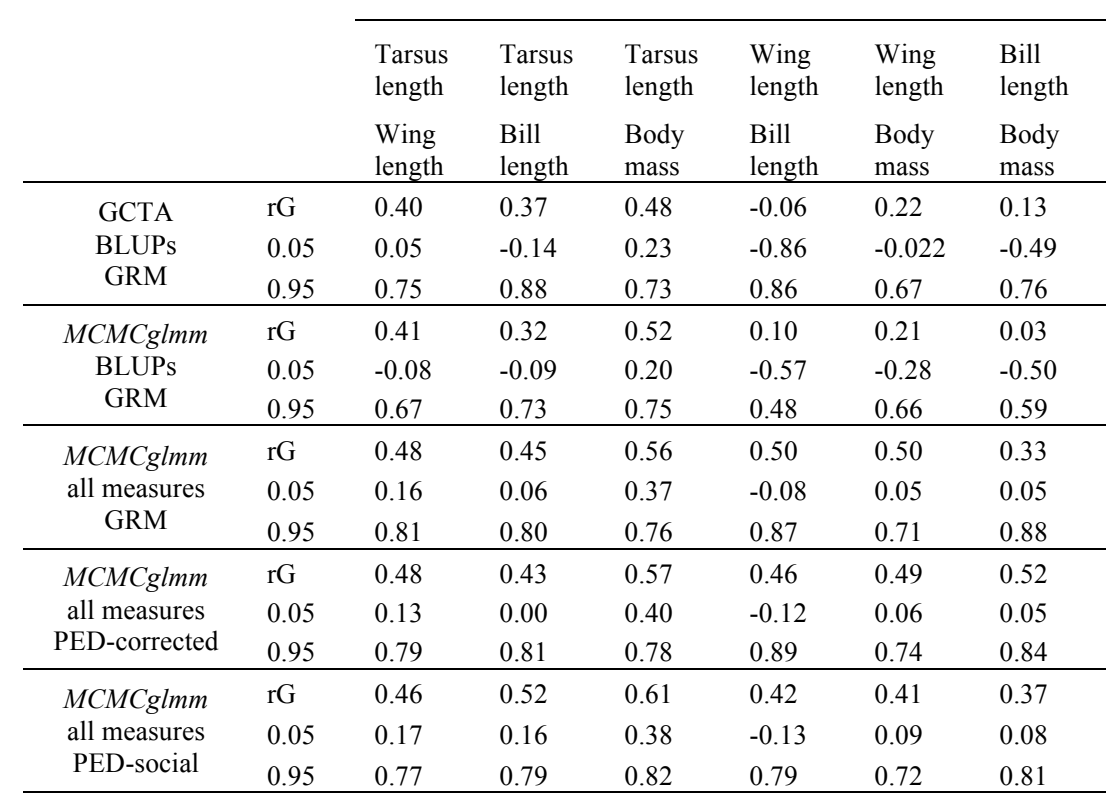


879 Table 4. Chromosome partitioning of the heritability estimated for the four phenotypic traits using GCTA. Partitioning was 880 achieved either i) fitting all the chromosomes simultaneously or ii) considering one chromosome versus all of the other 881 autosomes, iteratively for each chromosome. Several micro-chromosomes were grouped (22, 27, 28, 25LG1, 25LG2, 882 LGE22) and referred as "micros". "na" refers to the sets of chromosomes that have been removed to enable the 883 convergence of the model fitting simultaneously all the chromosomes (see methods). The underlined values do not 884 include 0 in their standard error intervals.

885 


\begin{tabular}{|c|c|c|c|c|c|c|c|c|c|c|c|c|c|c|c|c|c|c|}
\hline \multirow{3}{*}{$\begin{array}{c}\text { Chrom } \\
\text { osome } \\
\text { name }\end{array}$} & \multirow{3}{*}{ SNPs } & \multirow{3}{*}{ Genes } & \multicolumn{4}{|c|}{ Tarsus } & \multicolumn{4}{|c|}{ Wing length } & \multicolumn{4}{|c|}{ Bill length } & \multicolumn{4}{|c|}{ Body mass } \\
\hline & & & \multicolumn{2}{|c|}{$\begin{array}{c}\text { All } \\
\text { chromosomes } \\
\text { simultaneously }\end{array}$} & \multicolumn{2}{|c|}{$\begin{array}{c}\text { One } \\
\text { chromosome } \\
\text { versus the other, } \\
\text { iteratively }\end{array}$} & \multicolumn{2}{|c|}{$\begin{array}{c}\text { All } \\
\text { chromosomes } \\
\text { simultaneously }\end{array}$} & \multicolumn{2}{|c|}{$\begin{array}{c}\text { One } \\
\text { chromosome } \\
\text { versus the other, } \\
\text { iteratively }\end{array}$} & \multicolumn{2}{|c|}{$\begin{array}{c}\text { All } \\
\text { chromosomes } \\
\text { simultaneously }\end{array}$} & \multicolumn{2}{|c|}{$\begin{array}{c}\text { One } \\
\text { chromosome } \\
\text { versus the other, } \\
\text { iteratively }\end{array}$} & \multicolumn{2}{|c|}{$\begin{array}{c}\text { All } \\
\text { chromosomes } \\
\text { simultaneously }\end{array}$} & \multicolumn{2}{|c|}{$\begin{array}{c}\text { One } \\
\text { chromosome } \\
\text { versus the other, } \\
\text { iteratively }\end{array}$} \\
\hline & & & Media & SE & Media & SE & Media & $\mathrm{SE}$ & Media & SE & Media & SE & Media & SE & Media & SE & Media & SE \\
\hline 1 & 4784 & 1131 & 0 & 0.16 & 0 & 0.14 & 0.01 & 0.16 & 0.01 & 0.14 & 0 & 0.15 & 0 & 0.13 & 0.03 & 0.16 & 0.07 & 0.14 \\
\hline 2 & 5523 & 1287 & 0.06 & 0.17 & 0.01 & 0.15 & 0 & 0.17 & 0 & 0.16 & 0 & 0.16 & 0 & 0.15 & 0 & 0.19 & 0 & 0.16 \\
\hline 3 & 4383 & 1096 & 0.17 & 0.16 & 0.21 & 0.14 & 0 & 0.15 & 0 & 0.14 & 0.03 & 0.16 & 0.02 & 0.14 & 0.07 & 0.15 & 0.08 & 0.13 \\
\hline 4 & 2869 & 736 & 0 & 0.13 & 0 & 0.11 & 0.05 & 0.13 & 0.16 & 0.12 & 0.06 & 0.13 & 0.12 & 0.12 & 0.01 & 0.13 & 0.04 & 0.11 \\
\hline $4 \mathrm{~A}$ & 1428 & 343 & 0 & 0.09 & 0 & 0.08 & 0 & 0.1 & 0 & 0.09 & 0.05 & 0.1 & 0.11 & 0.09 & 0.01 & 0.09 & 0.01 & 0.08 \\
\hline $1 \mathrm{~A}$ & $\begin{array}{l}1420 \\
2638\end{array}$ & 853 & 0.21 & 0.13 & 0.24 & 0.11 & 0 & 0.12 & 0 & 0.11 & 0.13 & 0.13 & $\begin{array}{l}0.11 \\
0.17\end{array}$ & 0.12 & 0.04 & 0.12 & 0.06 & 0.11 \\
\hline 5 & 2902 & 940 & 0.04 & 0.12 & 0 & 0.1 & 0 & 0.12 & 0 & 0.11 & 0 & 0.13 & 0 & 0.11 & 0.09 & 0.13 & 0.1 & 0.11 \\
\hline 6 & 1818 & 513 & 0.04 & 0.11 & 0.11 & 0.1 & 0.01 & 0.1 & 0 & 0.09 & 0.01 & 0.1 & 0.07 & 0.09 & 0.12 & 0.11 & 0.17 & 0.1 \\
\hline 7 & 1907 & 488 & 0.06 & 0.11 & 0.21 & 0.1 & 0.01 & 0.11 & 0.06 & 0.1 & 0 & 0.12 & 0 & 0.1 & 0.07 & 0.11 & 0.11 & 0.1 \\
\hline 8 & 1718 & 492 & 0.04 & 0.1 & 0.11 & 0.09 & 0 & 0.1 & 0 & 0.09 & 0 & 0.11 & 0 & 0.1 & 0.01 & 0.1 & 0.04 & 0.1 \\
\hline 9 & 1689 & 434 & 0 & 0.11 & 0 & 0.09 & 0.09 & 0.1 & 0.16 & 0.1 & 0.03 & 0.1 & 0.05 & 0.09 & 0.02 & 0.1 & 0.01 & 0.09 \\
\hline 12 & 1573 & 343 & 0 & 0.1 & 0 & 0.08 & 0.04 & 0.09 & 0.04 & 0.08 & 0 & 0.1 & 0.01 & 0.08 & 0 & 0.08 & 0 & 0.09 \\
\hline 13 & 1371 & 322 & 0.03 & 0.08 & 0.08 & 0.08 & 0.01 & 0.08 & 0.02 & 0.08 & 0 & 0.09 & 0.03 & 0.08 & 0 & 0.09 & 0.08 & 0.08 \\
\hline 10 & 1177 & 394 & 0.07 & 0.09 & 0.1 & 0.08 & 0 & 0.08 & 0 & 0.07 & na & na & 0.14 & 0.08 & 0 & 0.1 & 0 & 0.07 \\
\hline 11 & 1309 & 364 & 0.03 & 0.09 & 0.12 & 0.09 & 0.05 & 0.09 & 0.11 & 0.08 & na & na & 0.02 & 0.08 & 0.08 & 0.09 & 0.01 & 0.08 \\
\hline 14 & 1364 & 396 & 0.05 & 0.09 & 0.08 & 0.08 & 0 & 0.08 & 0 & 0.08 & na & na & 0.04 & 0.08 & 0 & 0.09 & 0 & 0.08 \\
\hline 15 & 1004 & 358 & 0 & 0.08 & 0 & 0.07 & 0 & 0.08 & 0 & 0.07 & na & na & 0 & 0.08 & 0.03 & 0.08 & 0.01 & 0.07 \\
\hline 17 & 974 & 277 & 0.02 & 0.08 & 0.16 & 0.08 & 0 & 0.09 & 0 & 0.08 & na & na & 0.07 & 0.07 & 0.03 & 0.08 & 0.05 & 0.07 \\
\hline 18 & 1114 & 318 & $\begin{array}{c}0.02 \\
0\end{array}$ & 0.09 & 0 & 0.08 & 0 & 0.09 & 0 & 0.08 & na & na & $\begin{array}{c}.0 \\
0\end{array}$ & 0.08 & 0 & 0.08 & 0 & 0.07 \\
\hline 19 & 849 & 311 & 0 & 0.07 & 0 & 0.06 & 0.05 & 0.08 & 0.1 & 0.07 & na & na & 0.01 & 0.06 & 0.01 & 0.08 & 0.1 & 0.08 \\
\hline 21 & 632 & 260 & 0 & 0.07 & 0 & 0.06 & 0 & 0.07 & $\begin{array}{c}.1 \\
0\end{array}$ & 0.06 & na & na & 0 & 0.07 & 0 & 0.07 & $\begin{array}{c}0.1 \\
0\end{array}$ & 0.07 \\
\hline 24 & 846 & 175 & 0.02 & 0.08 & 0.04 & 0.07 & 0.02 & 0.07 & 0.08 & 0.07 & na & na & 0 & 0.07 & 0 & 0.08 & 0 & 0.07 \\
\hline 20 & 1301 & 341 & 0 & 0.09 & 0 & 0.08 & 0 & 0.09 & 0 & 0.08 & na & na & 0.17 & 0.09 & na & na & 0.01 & 0.07 \\
\hline 26 & 802 & 262 & 0 & 0.07 & 0 & 0.06 & 0.02 & 0.07 & 0.04 & 0.07 & na & na & 0.01 & 0.06 & na & na & 0 & 0.07 \\
\hline 23 & 521 & 232 & 0 & 0.07 & 0 & 0.06 & na & na & 0 & 0.06 & na & na & 0.07 & 0.06 & na & na & 0 & 0.06 \\
\hline micros & 1368 & 874 & 0 & 0.1 & 0.02 & 0.09 & na & na & 0.05 & 0.08 & na & na & 0 & 0.09 & na & na & 0 & 0.09 \\
\hline
\end{tabular}


889

890

891

892

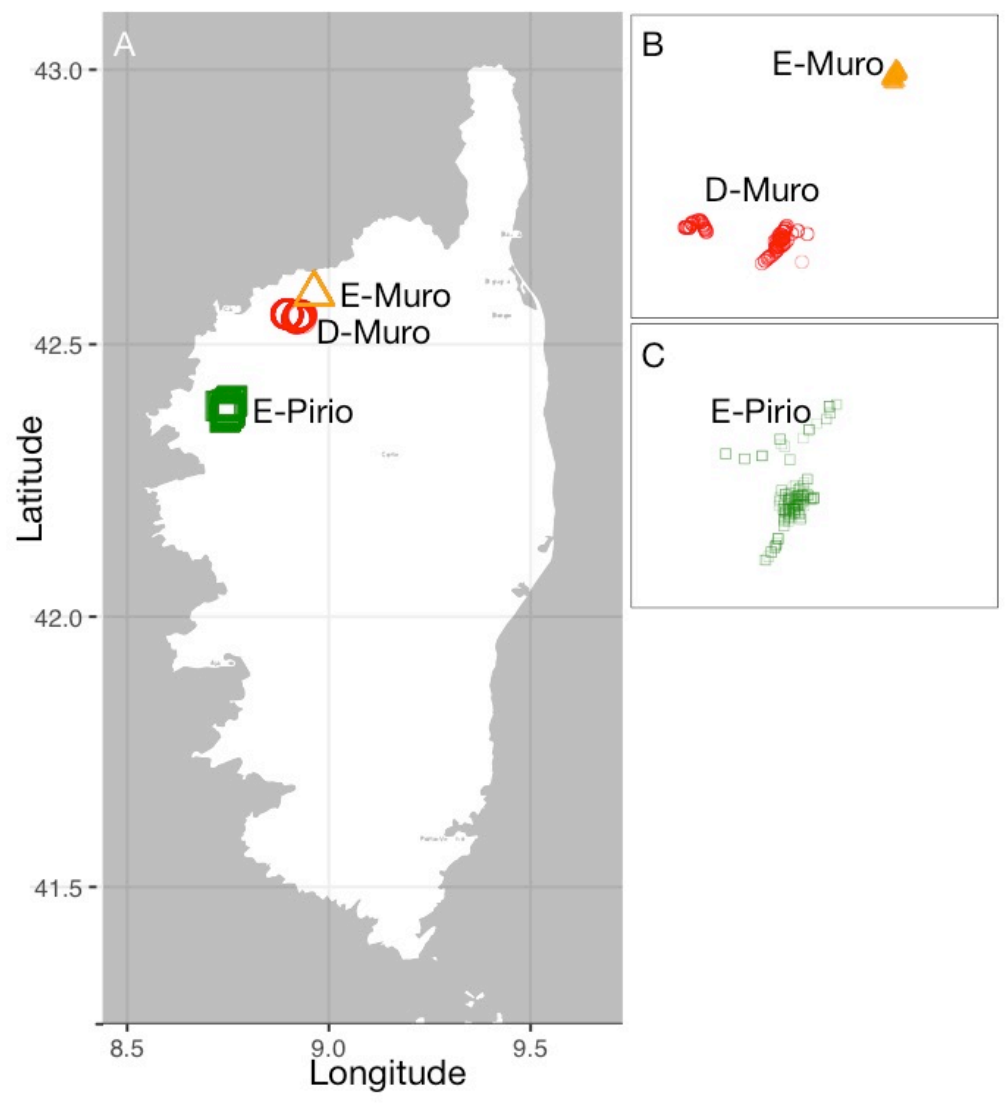

Figure 1. 


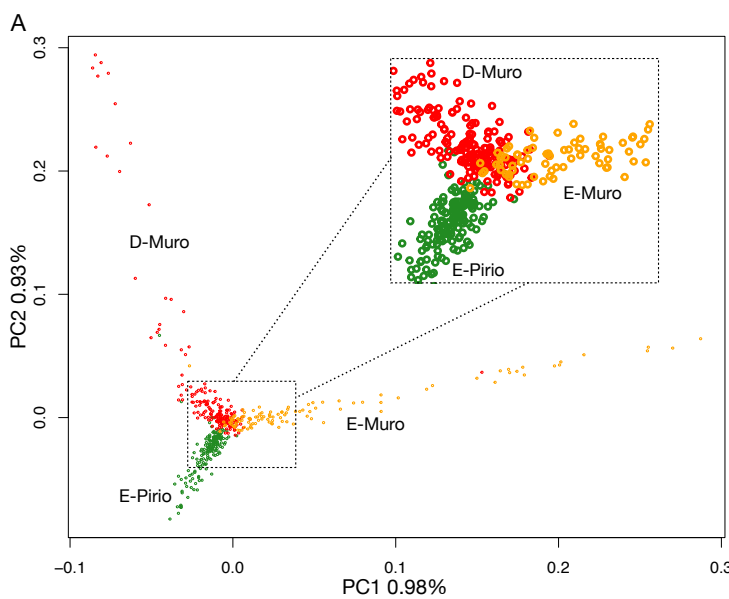

C

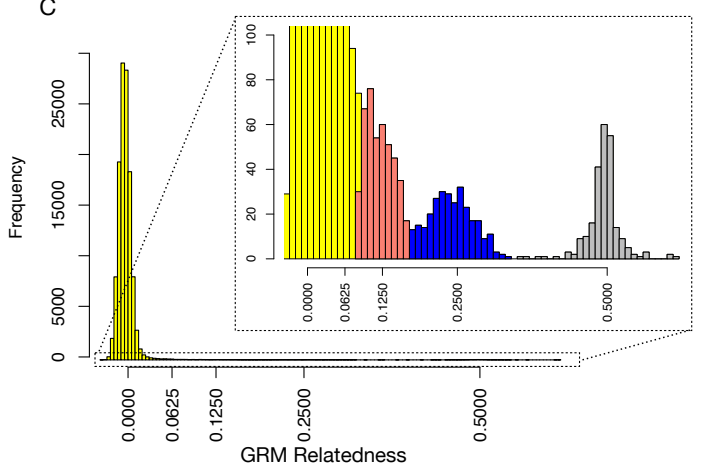

$894 \quad$ Figure 2.

895

896
893
B

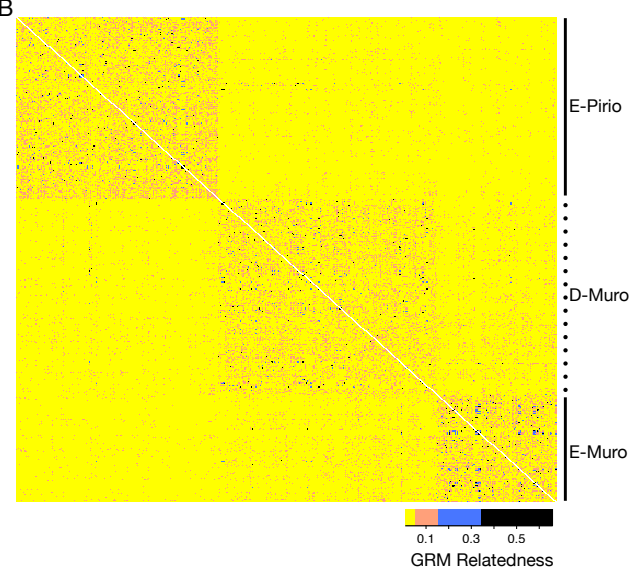

D

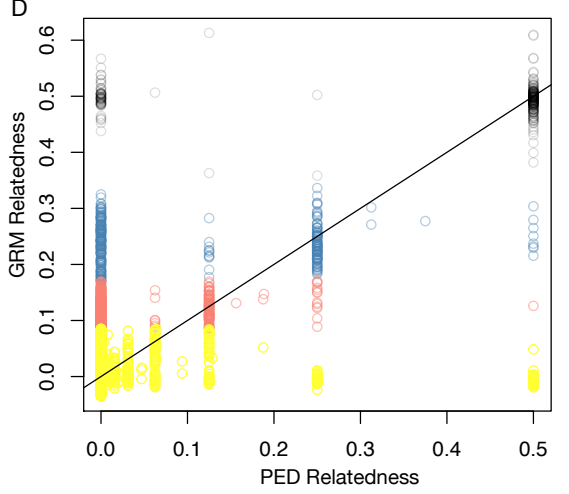



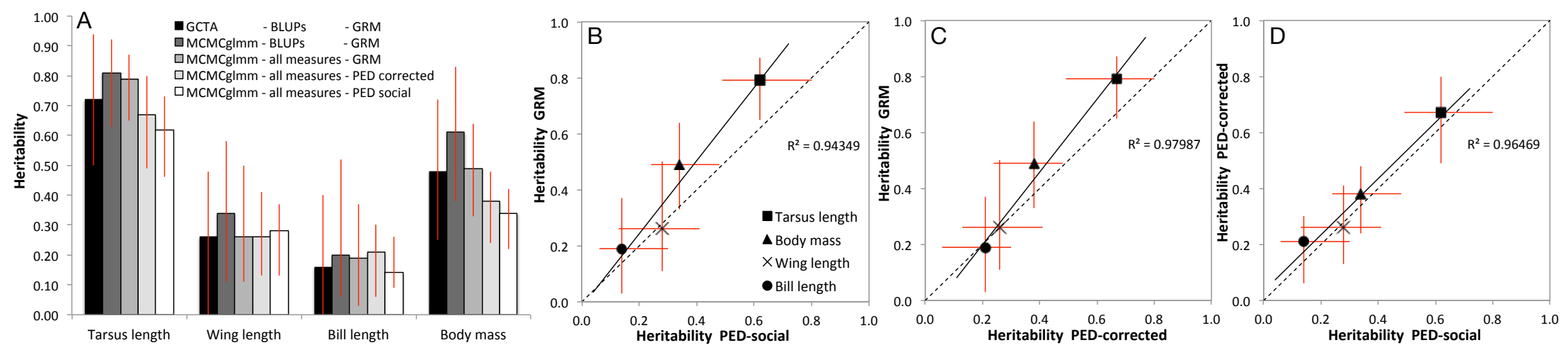

Figure 3. 
899
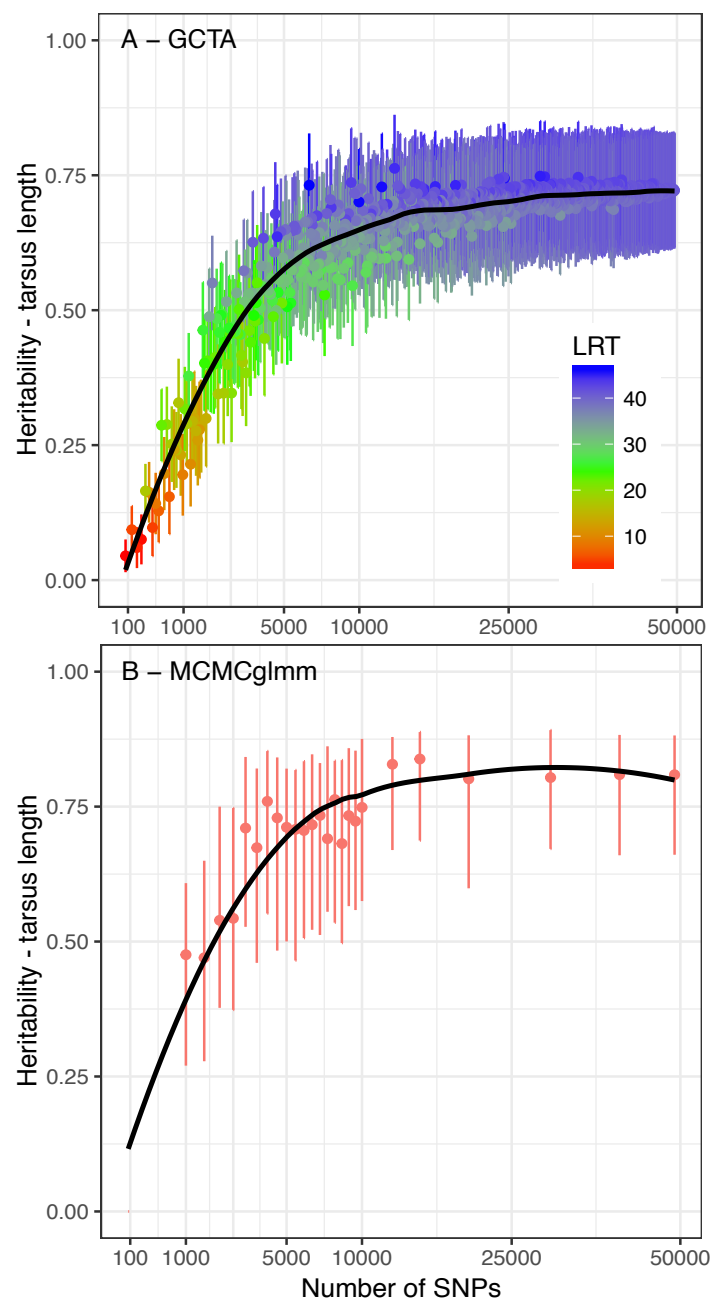

900

901

Figure 4.

902 


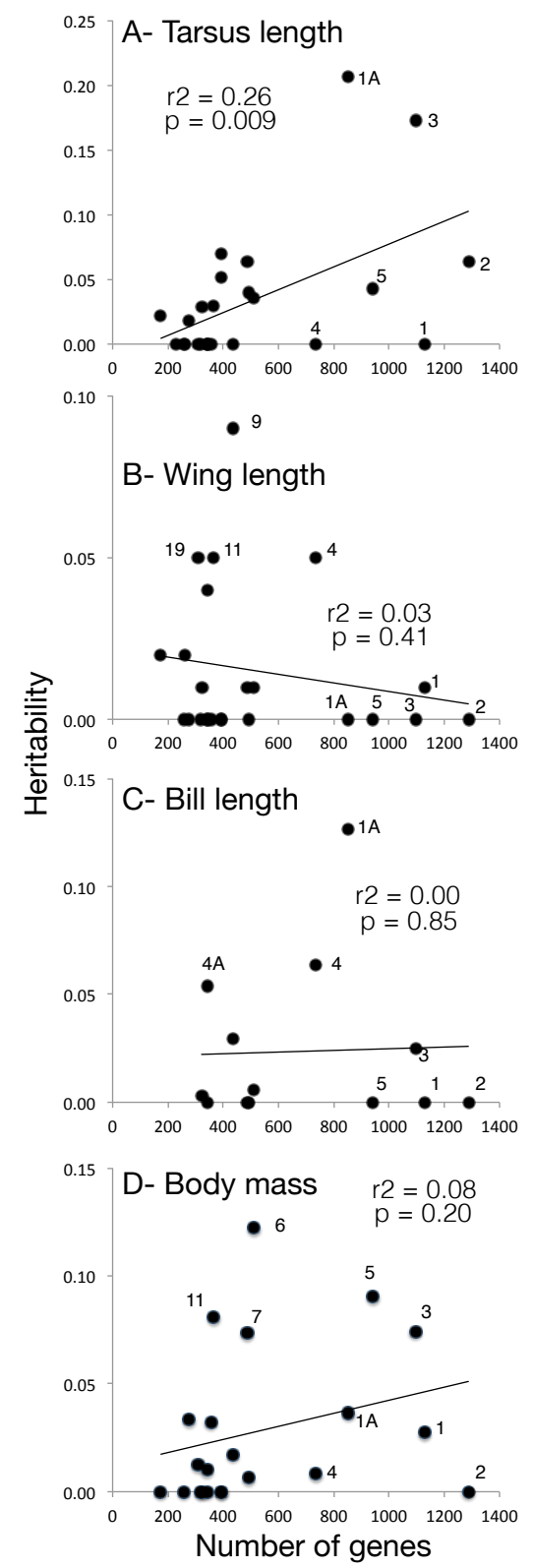

903

$904 \quad$ Figure 5.

905 
906 Supplementary material

907 Supplementary material 1. Box plots showing sex- and site-specific median and

908 variation in tarsus length, wing length, bill length and body mass for the 494

909 genotyped individuals. The first four letters code for the site and the last letter

910 codes for the sex (F: female; M: male).

911 Supplementary material 2 . Social pedigree characteristics.

912 Supplementary material 3. Heterozygosity $(\mathrm{He})$ in E-Piro, D-Muro and E-Muro

913 and FST among the three sites (FST above diagonal and p-value below the 914 diagonal).

915 Supplementary material 4. Variance estimates associated to the other random 916 factors.

917

918 

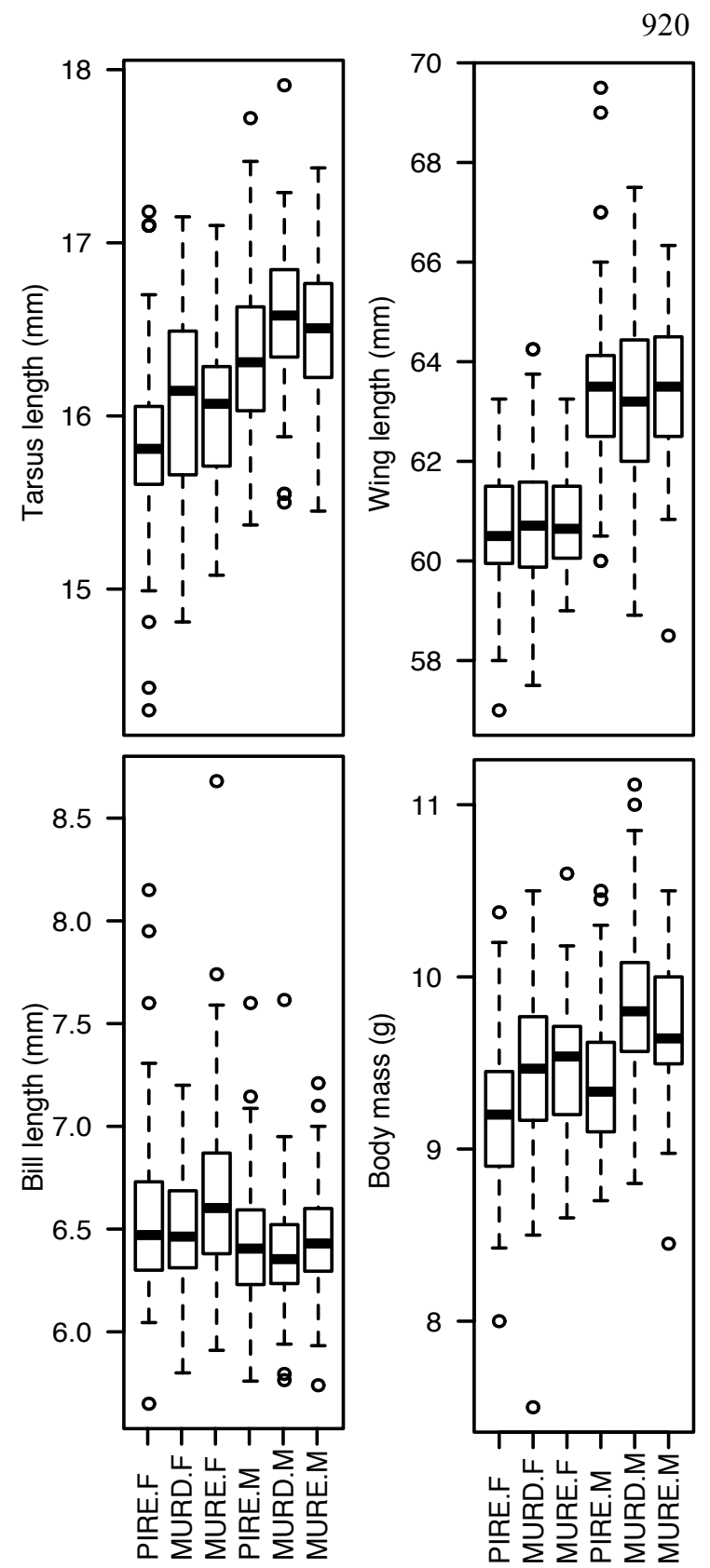
924 Supplementary material 2.

$\begin{array}{lcccccc} & \text { Maternities } & \text { Paternities } & \text { Full-sibs } & \text { Maternal half-sibs } & \text { Paternal half-sibs } & \text { Max Depth } \\ \text { Pooled sites } & 333 & 333 & 88 & 47 & 35 & 14 \\ \text { E-Pirio } & 115 & 115 & 27 & 15 & 9 & 14 \\ \text { E-Muro } & 117 & 117 & 23 & 18 & 17 & 7 \\ \text { D-Muro } & 116 & 116 & 34 & 13 & 9 & 6\end{array}$

929 Supplementary material 3.

\begin{tabular}{lllll}
\cline { 2 - 4 } & \multicolumn{4}{l}{$F_{\text {ST }}$} \\
\cline { 2 - 5 } & He & E-Piro & D-Muro & E-Muro \\
\hline E-Piro & 0.205 & - & 0.006 & 0.008 \\
D-Muro & 0.205 & $p<0.001$ & -- & 0.007 \\
E-Muro & 0.204 & $p<0.001$ & $p<0.001$ & -- \\
\hline
\end{tabular}

930 


\section{Supplementary material 4.}

932 Variance associated to the permanent environment effect.

\begin{tabular}{llcccccccccccc} 
& & \multicolumn{3}{c}{ Tarsus } & \multicolumn{4}{c}{ Wing } & \multicolumn{4}{c}{ Bill } & \multicolumn{3}{c}{ Mass } \\
& & & Vpe & $95 \% \mathrm{Cl}$ & Vpe & $95 \% \mathrm{Cl}$ & Vpe & $95 \% \mathrm{Cl}$ & Vpe & $95 \% \mathrm{Cl}$ \\
\cline { 3 - 13 } GRM & Pooled & 0.04 & 0.01 & 0.07 & 0.65 & 0.4 & 1.09 & 0.03 & 0.01 & 0.05 & 0.02 & 0.01 & 0.06 \\
GRM & E-Pirio & 0.03 & 0.01 & 0.11 & 0.65 & 0.24 & 1.03 & 0.03 & 0.01 & 0.05 & 0.03 & 0.01 & 0.09 \\
GRM & D-Muro & 0.03 & 0.01 & 0.09 & 0.73 & 0.24 & 1.42 & 0.02 & 0 & 0.03 & 0.08 & 0.01 & 0.11 \\
GRM & E-Muro & 0.02 & 0.01 & 0.07 & 0.44 & 0.12 & 0.97 & 0.02 & 0.01 & 0.07 & 0.01 & 0 & 0.06 \\
PED-corrected & Pooled & 0.03 & 0.01 & 0.06 & 0.5 & 0.2 & 0.9 & 0.02 & 0.01 & 0.04 & 0.02 & 0.01 & 0.05 \\
PED-corrected & E-Pirio & 0.03 & 0.01 & 0.12 & 0.5 & 0.18 & 0.96 & 0.02 & 0 & 0.04 & 0.02 & 0.01 & 0.09 \\
PED-corrected & D-Muro & 0.02 & 0.01 & 0.1 & 0.38 & 0.12 & 1.1 & 0.01 & 0 & 0.03 & 0.02 & 0.01 & 0.08 \\
PED-corrected & E-Muro & 0.02 & 0.01 & 0.08 & 0.45 & 0.12 & 0.88 & 0.02 & 0.01 & 0.07 & 0.02 & 0.01 & 0.07
\end{tabular}

933

934 Variance associated to the observer effect.

\begin{tabular}{llccccccccccccc} 
& & \multicolumn{3}{c}{ Tarsus } & \multicolumn{4}{c}{ Wing } & \multicolumn{4}{c}{ Bill } & \multicolumn{4}{c}{ Mass } \\
& & & Vobs & $95 \% \mathrm{Cl}$ & Vobs & $95 \% \mathrm{Cl}$ & Vobs & $95 \% \mathrm{Cl}$ & Vobs & $95 \% \mathrm{Cl}$ \\
\hline GRM & Pooled & 0.01 & 0.01 & 0.02 & 0.23 & 0.12 & 0.52 & 0.05 & 0.02 & 0.1 & 0.01 & 0.01 & 0.03 \\
GRM & E-Pirio & 0.01 & 0 & 0.03 & 0.21 & 0.08 & 0.46 & 0.02 & 0.01 & 0.06 & 0.01 & 0 & 0.03 \\
GRM & D-Muro & 0.01 & 0.01 & 0.04 & 0.36 & 0.15 & 1.05 & 0.07 & 0.03 & 0.16 & 0.02 & 0.01 & 0.05 \\
GRM & E-Muro & 0.01 & 0.01 & 0.04 & 0.26 & 0.11 & 0.72 & 0.04 & 0.01 & 0.09 & 0.02 & 0.01 & 0.08 \\
PED-corrected & Pooled & 0.01 & 0.01 & 0.02 & 0.24 & 0.11 & 0.53 & 0.05 & 0.02 & 0.1 & 0.02 & 0.01 & 0.04 \\
PED-corrected & E-Pirio & 0.01 & 0.01 & 0.03 & 0.21 & 0.08 & 0.45 & 0.02 & 0.01 & 0.06 & 0.01 & 0 & 0.03 \\
PED-corrected & D-Muro & 0.01 & 0.01 & 0.04 & 0.35 & 0.15 & 0.97 & 0.07 & 0.03 & 0.17 & 0.01 & 0.01 & 0.05 \\
PED-corrected & E-Muro & 0.01 & 0.01 & 0.04 & 0.33 & 0.09 & 0.8 & 0.04 & 0.02 & 0.09 & 0.03 & 0.01 & 0.08
\end{tabular}

935

936

Variance associated to the residuals.

\begin{tabular}{lcccccccccccccc} 
& \multicolumn{4}{c}{} & \multicolumn{4}{c}{ Tarsus } & \multicolumn{4}{c}{ Wing } & \multicolumn{4}{c}{ Bill } & \multicolumn{4}{c}{ Mass } \\
& & & Vresiduals & $95 \% \mathrm{Cl}$ & Vresiduals & $95 \% \mathrm{Cl}$ & Vresiduals & $95 \% \mathrm{Cl}$ & Vresiduals & $95 \% \mathrm{Cl}$ \\
GRM & Pooled & 0.03 & 0.03 & 0.04 & 1.22 & 1.14 & 1.35 & 0.1 & 0.08 & 0.1 & 0.13 & 0.12 & 0.14 \\
GRM & E-Pirio & 0.03 & 0.03 & 0.04 & 1.68 & 1.46 & 1.95 & 0.11 & 0.09 & 0.13 & 0.14 & 0.13 & 0.17 \\
GRM & D-Muro & 0.03 & 0.03 & 0.04 & 0.9 & 0.77 & 1.04 & 0.06 & 0.05 & 0.07 & 0.14 & 0.12 & 0.16 \\
GRM & E-Muro & 0.02 & 0.02 & 0.03 & 1.12 & 0.97 & 1.39 & 0.09 & 0.07 & 0.11 & 0.09 & 0.07 & 0.1 \\
PED-corrected & Pooled & 0.03 & 0.03 & 0.04 & 1.26 & 1.13 & 1.35 & 0.09 & 0.08 & 0.1 & 0.13 & 0.12 & 0.14 \\
PED-corrected & E-Pirio & 0.03 & 0.03 & 0.04 & 1.62 & 1.42 & 1.93 & 0.11 & 0.09 & 0.13 & 0.14 & 0.13 & 0.17 \\
PED-corrected & D-Muro & 0.03 & 0.03 & 0.04 & 0.88 & 0.77 & 1.03 & 0.06 & 0.05 & 0.07 & 0.13 & 0.12 & 0.16 \\
PED-corrected & E-Muro & 0.02 & 0.02 & 0.03 & 1.18 & 1 & 1.39 & 0.09 & 0.07 & 0.11 & 0.09 & 0.07 & 0.11
\end{tabular}

